Успехи физ. мет. / Usp. Fiz. Met. 2012, т. 13, сс. 189-223 Оттиски доступны непосредственно от издателя Фотокопирование разрешено только в соответствии с лицензией (c) 2012 ИМФ (Институт металлофизики им. Г. В. Курдюмова НАН Украины)

Напечатано в Украине.

PACS numbers: 63.20.K-, 71.15.Ap, 71.20.Tx, 71.27.+a, 72.10.-d, 72.25.-b, 73.63.Fg

\title{
Self-Consistent Model of Strong Coupling Theory of Electron Correlations in Disordered Crystals
}

\author{
S. P. Repetsky ${ }^{1}$, O. V. Tretyak ${ }^{2}$, I. G. Vyshivanaya ${ }^{2}$, and V. V. Shastun ${ }^{1}$ \\ ${ }^{1}$ Department of Physics, Taras Shevchenko National University of Kyiv, \\ Volodymyrs'ka Str., 64, \\ 01033 Kyyiv, Ukraine \\ ${ }^{2}$ Institute of High Technologies, \\ Taras Shevchenko National University of Kyiv, \\ Academician Glushkov Ave., $4^{g}$, \\ 03022 Kyyiv, Ukraine
}

The method of the description of electron correlations in the disordered crystals based on a Hamiltonian of many-particle system as well as the diagram technique for calculation of Green's functions are developed. Electron states of system are featured within the scope of the self-consistent tight-binding multiband model. Processes of scattering of electrons on potentials of the ion cores of different kinds and oscillations of a crystal lattice are considered. The consecutive method for the account of the long-range Coulomb interaction of electrons at different sites of a lattice is offered. The cluster expansion for a density of states, a free energy and an electrical conductivity of the disordered system is obtained. As shown, the contributions of processes of scattering of elementary excitations on clusters decrease with increasing number of sites in a cluster according to some small parameter. Precision of calculation is defined by accuracy of a renormalization of vertex parts of mass operators of electron-electron and electron-phonon interactions as well as by small parameter of the cluster expansion. The nature of spin-dependent electron transport in carbon nanotubes with chromium atoms adsorbed on a surface is found out. As shown, the quantity of spin-dependent transport is related to the relative offset of energy levels of electrons (i.e. to the Coulomb gaps arising in a vicinity of the Fermi level) for different projections of a spin in an external magnetic field.

Розвинено методу опису електронних кореляцій у невпорядкованих кристалах, що базується на Гамільтоніяні багаточастинкової системи та діяграмній техніці для розрахунку Грінових функцій. Електронні стани системи описано в рамках самоузгодженого багатозонного моделю сильного зв'язку. Враховано процеси розсіяння електронів на потенціялах йонних кістяків різного сорту та коливаннях кристалічної гратниці. Запропоно- 
вано послідовну методу врахування далекосяжної Кульонової взаємодії електронів на різних вузлах гратниці. Одержано кластерне розвинення для густини станів, вільної енергії та електропровідности невпорядкованої системи. Показано, що внески процесів розсіяння елементарних збуджень на кластерах зменшуються зі збільшенням числа вузлів у кластері за деяким малим параметром. Точність розрахунку визначається точністю перенормування вершинних частин масових операторів електронелектронної і електрон-фононної взаємодій та малим параметром кластерного розвинення. 3'ясовано природу спін-залежного електронного транспорту вуглецевих нанорурок з атомами хрому, адсорбованими на поверхні. Показано, що величина спін-залежного транспорту пов'язана з відносним зсувом у зовнішньому магнетному полі енергетичних рівнів електронів (Кульоновими щілинами, що виникають в області рівня Фермі) для різних проєкцій спіну.

Развит метод описания электронных корреляций в неупорядоченных кристаллах, основанный на гамильтониане многочастичной системы и диаграммной технике для расчёта функций Грина. Электронные состояния системы описаны в рамках самосогласованной многозонной модели сильной связи. Учтены процессы рассеяния электронов на потенциалах ионных остовов разного сорта и колебаниях кристаллической решётки. Предложен последовательный метод учёта дальнодействующего кулоновского взаимодействия электронов на разных узлах решётки. Получено кластерное разложение для плотности состояний, свободной энергии и электропроводности неупорядоченной системы. Показано, что вклады процессов рассеяния элементарных возбуждений на кластерах уменьшаются с увеличением числа узлов в кластере в соответствии с некоторым малым параметром. Точность расчёта определяется точностью перенормировки вершинных частей массовых операторов электрон-электронного и электрон-фононного взаимодействий и малым параметром кластерного разложения. Выяснена природа спин-зависимого электронного транспорта углеродных нанотрубок с атомами хрома, адсорбированными на поверхности. Показано, что величина спин-зависимого транспорта связана с относительным сдвигом во внешнем магнитном поле энергетических уровней электронов (кулоновскими щелями, возникающими в области уровня Ферми) для разных проекций спина.

Key words: self-consistent tight-binding model, electron correlations, carbon nanotubes doped with $\mathrm{Cr}$ atoms, energy spectrum of electrons and phonons, electroconductivity, spin polarization of electric current.

(Received March 15, 2012)

\section{INTRODUCTION}

Progress in describing of disordered systems is strongly concerned with development of electron theory. Substitutional alloys are best described among disordered systems. Traditional knowledge about 
physical properties of alloys is based on the Born approximation of the scattering theory. However, this approach obviously cannot be applied in case of a large scattering potential difference of components that holds for the description of alloys with simple, transition and rareearth elements. The same difficulty relates the pseudopotential method [1]. Because of non-local nature of pseudopotential, the problem of pseudopotential transferability exists. It is impossible to use nuclear potentials determined by the properties of some systems to describe other systems. Due to using the theory of Vanderbilt ultra-soft potentials [2, 3] and method of projector-augmented waves proposed by Blochl $[4,5]$, fundamental progress in investigations of electronic structure and properties of the system has been achieved. Significant success in the study of electronic structure and properties of the systems was achieved recently because of the use of ultra-soft pseudopotential Vanderbilt $[2,3]$ and the method of projector-augmented waves within the density functional theory proposed by Blochl [4, 5]. This approach was developed further because of use of the generalized gradient approximation in density functional theory of multi-electron systems developed in Perdew's works [6-10]. In projector-augmented waves approach, the wave function of valence states of electron (allelectron orbital) is expressed by using the conversion through the pseudoorbital. Pseudoorbital expands to pseudo partial waves in the augment area. Even so, all-electron orbital in the same area is expanded with the same coefficients via partial waves described by Kohn-Sham equation. Expression for pseudo-Hamiltonian, which we have in equation for pseudo-wave function, is derived by minimizing the total energy functional. Using this equation and expanding pseudoorbital into plane waves, we can derive set of equations for expansion coefficients. With this set, it is possible to get electron energy spectrum, wave functions, and value of the total energy functional. As shown in [10], there is a way to use this method for describing the electron structure of crystals, using VASP program package. Using cluster methods of calculation and GAUSSIAN program package, this approach could be used for description molecule electronic structure.

It should be noted that simple effective calculation method of electronic structure and properties for big molecules had been proposed recently in [10-17]. This method is based on tight-binding model and density functional theory, which includes long-range Coulomb interaction of electrons at different sites of crystal lattice. Long-range Coulomb interaction of electrons at different sites is described in the local density approximation.

However, mentioned methods [6-17] are used only for description of ideal ordered crystals and molecules.

In disordered crystals, effects associated with localized electron states and lattice vibrations occur. They cannot be described with a 
model of an ideal crystal. In this regard, other approaches are developed too.

Essential achievement in description of properties of disordered systems is connected with application of tight-binding model in the multielectron scattering, including approximation of coherent potential. Starting from Slater-Koster works [18], there was wide use of the tight-binding model in electronic-structure calculations and in description of ideal crystals properties [19]. Later, it was generalized for the case of disordered systems.

In Refs [20, 21], method of describing magnetic alloys electronic structure based on functional density theory is proposed. The effective potential in Kohn-Sham equation [22, 23] consists of atomic potential and Pauli addition, which is expressed through magnetic field induction. Atomic potential and induction of magnetic field are expressed through variational derivative of exchange-correlation energy by electron density and magnetization, respectively. Calculations of electronic structure of magnetic alloy are based on already mentioned effective mass potentials using self-consistent Korringa-Kohn-Rostoker approximation - the coherent potential, but more developed in [2426]. In Ref. [20], a method is proposed for calculating the parameters of interatomic pair correlations due to the pair mixing potential, which is expressed through the second derivative of the thermodynamic potential of the alloy concentration [27]. This thermodynamic potential is calculated within the one-site coherent potential approximation. It should be noted that the methods developed in [18-20, 24-26] do not include long-range Coulomb interaction of electrons at different lattice sites.

For calculations of energy spectrum, free energy and electroconductivity of disordered crystals in our work, multi-scattering theory based on Green's functions is developed. Electron correlations in crystal are described in multiband tight-binding model. It includes recalculation of wave functions and atomic potentials with taking into account the electron density redistribution because of atomic interaction. The model includes long-range Coulomb interaction of electrons on different sites of crystal lattice. The wave functions of noninteracting atoms are calculated with Kohn-Sham equation using the Perdew potentials [6-10]. Electron scattering processes on the ion core potentials of different kinds and on vibrations of crystal lattice are taken into account. Calculations of two-time Green's functions are based on temperature Green's functions [28]. This uses a known relation between spectral representation for two-time and temperature Green's function [29].

Calculation of two-time Green's function of disordered crystal is based on diagram techniques, which are analogous to diagram technique for homogeneous system [29]. Set of equations for temperature Green's function, expressions for both free energy and electroconduc- 
tivity of solids are derived. Accuracy of the energy spectrum, free energy and crystal conductivity calculations is based on renormalization of vertex parts of the electron-electron and electron-phonon mass operators. Calculations based on this method for energy spectrum, free energy, conductivity and spin-dependent transport of nanotubes doped with $\mathrm{Cr}$ atoms were performed.

\section{HAMILTONIAN FOR SYSTEM OF ELECTRONS AND PHONONS IN DISORDERED CRYSTALS}

Hamiltonian of disordered system (alloy, disordered semiconductor) consists of Hamiltonian of electrons in the external ion field, the Hamiltonian of electron-electron interaction, the Hamiltonian of ions, and the Hamiltonian of electron-ion interaction. Motion of ion subsystem is reduced to the ions' oscillations near equilibrium positions under the influence of ions' interaction forces and their indirect interaction through electrons. Within the Wannier representation, the system Hamiltonian is as follows [28]:

$$
H=H_{0}+H_{\text {int }},
$$

where the zero-order Hamiltonian,

$$
H_{0}=\Phi_{0}+H_{f 0}+H_{e 0},
$$

consists of noninteracting electrons' subsystem Hamiltonian in an external field of the $A$ kind ions,

$$
H_{e 0}=\sum_{\substack{n_{1} i_{1} \gamma_{1} \\ n_{2} i_{2} \gamma_{2}}} h_{n_{1} \gamma_{1} \gamma_{1}, n_{2} i_{2} \gamma_{2}}^{(0)} a_{n_{1} i_{1} \gamma_{1}}^{+} a_{n_{2} i_{2} \gamma_{2}},
$$

Hamiltonian of subsystem of the $A$ kind ions,

$$
H_{f 0}=\sum_{n i \alpha} \frac{P_{\alpha}^{2}\left(\begin{array}{c}
n \\
i
\end{array}\right)}{2 M_{A}}+\frac{1}{2} \sum_{\substack{n_{1} i_{1} \alpha_{1} \\
n_{2} i_{2} \alpha_{2}}} \Phi_{\alpha_{1} \alpha_{2}}^{(0)}\left(\begin{array}{cc}
n_{1} & n_{2} \\
i_{1} & i_{2}
\end{array}\right) u_{\alpha_{1}}\left(\begin{array}{c}
n_{1} \\
i_{1}
\end{array}\right) u_{\alpha_{2}}\left(\begin{array}{c}
n_{2} \\
i_{2}
\end{array}\right),
$$

and the electrostatic-interaction energy of ions at equilibrium positions, $\Phi_{0}$.

Perturbation Hamiltonian in (1),

$$
H_{\mathrm{int}}=H_{e i}+H_{e f}+H_{e e}+H_{f i}+H_{f f} \text {, }
$$

consists of electron-ion interaction Hamiltonian, 
194 S. P. REPETSKY, O. V. TRETYAK, I. G. VYSHIVANAYA, and V. V. SHASTUN

$$
H_{e i}=\sum_{\substack{n_{1} i_{1} \gamma_{1} \\ n_{2} i_{2} \gamma_{2}}} w_{n_{1} i_{1} \gamma_{1}, n_{2} i_{2} \gamma_{2}} a_{n_{1} i_{1} \gamma_{1}}^{+} a_{n_{2} i_{2} \gamma_{2}},
$$

Hamiltonian of electrons' interactions with oscillations of crystal lattice (electron-phonon interaction),

$$
H_{e f}=\sum_{\substack{n_{1} i_{1} \gamma_{1} \\ n_{2} i_{2} \gamma_{2}}} v_{n_{1} i_{1} \gamma_{1}, n_{2} i_{2} \gamma_{2}}^{\prime} a_{n_{1} \dot{i}_{1} \gamma_{1}}^{+} a_{n_{2} i_{2} \gamma_{2}},
$$

electron-electron pair interaction Hamiltonian,

$$
H_{e e}=\frac{1}{2} \sum_{\substack{n_{1} i_{1} \gamma_{1} \\ n_{2} i_{2} \gamma_{2} \\ n_{3} i_{3} \gamma_{3} \\ n_{4} i_{4} \gamma_{4}}} v_{n_{3} i_{3} \gamma_{3}, n_{4} i_{4} \gamma_{4}}^{(2) n_{1} i_{1} \gamma_{1}, n_{2} i_{2} \gamma_{2}} a_{n_{1} i_{1} \gamma_{1}}^{+} a_{n_{2} i_{2} \gamma_{2}}^{+} a_{n_{3} i_{3} \gamma_{3}} a_{n_{4} i_{4} \gamma_{4}},
$$

component of ion-ion interaction Hamiltonian caused by the presence of different kinds of atoms (phonon-impurity interaction),

$$
\begin{aligned}
H_{f i} & =\frac{1}{2} \sum_{\substack{n_{1} i_{1} \alpha_{1} \\
n_{2} i_{2} \alpha_{2}}} \Delta M_{\alpha_{1} \alpha_{2}}^{-1}\left(\begin{array}{cc}
n_{1} & n_{2} \\
i_{1} & i_{2}
\end{array}\right) P_{\alpha_{1}}\left(\begin{array}{c}
n_{1} \\
i_{1}
\end{array}\right) P_{\alpha_{2}}\left(\begin{array}{c}
n_{2} \\
i_{2}
\end{array}\right)+ \\
& +\frac{1}{2} \sum_{\substack{n_{1} i_{1} \alpha_{1} \\
n_{2} i_{2} \alpha_{2}}} \Delta \Phi_{\alpha_{1} \alpha_{2}}\left(\begin{array}{cc}
n_{1} & n_{2} \\
i_{1} & i_{2}
\end{array}\right) u_{\alpha_{1}}\left(\begin{array}{c}
n_{1} \\
i_{1}
\end{array}\right) u_{\alpha_{2}}\left(\begin{array}{c}
n_{2} \\
i_{2}
\end{array}\right),
\end{aligned}
$$

where

$$
\begin{gathered}
\Delta M_{\alpha_{1} \alpha_{2}}^{-1}\left(\begin{array}{cc}
n_{1} & n_{2} \\
i_{1} & i_{2}
\end{array}\right)=\left(\frac{1}{M_{n_{1} i_{1}}}-\frac{1}{M_{A}}\right) \delta_{n_{1} n_{2}} \delta_{i_{1} i_{2}} \delta_{\lambda_{1} \lambda_{2}}, \\
\Delta \Phi_{\alpha_{1} \alpha_{2}}\left(\begin{array}{cc}
n_{1} & n_{2} \\
i_{1} & i_{2}
\end{array}\right)=\Phi_{\alpha_{1} \alpha_{2}}\left(\begin{array}{cc}
n_{1} & n_{2} \\
i_{1} & i_{2}
\end{array}\right)-\Phi_{\alpha_{1} \alpha_{2}}^{(0)}\left(\begin{array}{cc}
n_{1} & n_{2} \\
i_{1} & i_{2}
\end{array}\right),
\end{gathered}
$$

and anharmonic part of ion-ion interaction Hamiltonian (phononphonon interaction),

$$
H_{f f}=\frac{1}{3 !} \sum_{\substack{n_{1} i_{1} \alpha_{1} \\
n_{2} i_{2} \alpha_{2} \\
n_{3} i_{3} \alpha_{3}}} \Phi_{\alpha_{1} \alpha_{2} \alpha_{3}}^{(0)}\left(\begin{array}{ccc}
n_{1} & n_{2} & n_{3} \\
i_{1} & i_{2} & i_{3}
\end{array}\right) u_{\alpha_{1}}\left(\begin{array}{c}
n_{1} \\
i_{1}
\end{array}\right) u_{\alpha_{2}}\left(\begin{array}{c}
n_{2} \\
i_{2}
\end{array}\right) u_{\alpha_{3}}\left(\begin{array}{c}
n_{3} \\
i_{3}
\end{array}\right) .
$$

In expression of ion-ion interaction Hamiltonian (10), only anharmonic terms of third order were taken into account.

In expressions written above, $a_{n i \gamma}^{+}, a_{n i \gamma}$ are the creation and destruc- 
tion operators in the state described by the Wannier function, $\varphi_{n i \gamma}(\xi)=\langle\xi \mid n i \gamma\rangle, \xi=(\mathbf{r}, \sigma)$. State index $\gamma$ includes a quantum number, $\sigma=1 / 2,-1 / 2$, which defines value of spin projection on $Z$ axis, and a set of other quantum numbers describing spatial movements of electrons. Here, $n$ is the number of primitive cell, $i$ - the sublattice-site number in primitive cell, $r$ - the radius vector of electron, $h_{n_{1} i_{1}, n_{2} i_{2} \gamma_{2}}^{()_{2}}-$ matrix elements of Hamiltonian of electrons in an external field of the $A$ kind ion cores, $\mathbf{u}\left(\begin{array}{c}n \\ i\end{array}\right)$-the atom displacement operator at the site $(n i) ; P_{\alpha}\left(\begin{array}{l}n \\ i\end{array}\right)-$ the operator of $\alpha$-projection of atom momentum onto orthogonal axes, $\Phi_{\alpha_{1} \alpha_{2}}^{(0)}\left(\begin{array}{cc}n_{1} & n_{2} \\ i_{1} & i_{2}\end{array}\right), \Phi_{\alpha_{1} \alpha_{2} \alpha_{3}}^{(0)}\left(\begin{array}{ccc}n_{1} & n_{2} & n_{3} \\ i_{1} & i_{2} & i_{3}\end{array}\right)$-force constants related to potential energy of the $A$ kind ion interactions.

Potential energy operator of electron in a field of different-kind ion cores can be expressed as

$$
V(\mathbf{r})=\sum_{n i} v^{n i}\left(\mathbf{r}-\mathbf{r}_{n i}^{\prime}\right), r_{n i}^{\prime}=r_{n i}+u^{s}\left(\begin{array}{c}
n \\
i
\end{array}\right)+u\left(\begin{array}{c}
n \\
i
\end{array}\right),
$$

where $\mathbf{r}$-electron radius vector, $\mathbf{r}_{n i}=\mathbf{r}_{n}+\boldsymbol{\rho}_{i}$-radius vector of atom equilibrium position at the site $(n i)$ in a crystal lattice, $\mathbf{u}^{s}\left(\begin{array}{l}n \\ i\end{array}\right)$-vector of atom static displacement from equilibrium position at site ( $n i$ ). Random addition to matrix element of one-electron Hamiltonian of a pure crystal caused by impurity presence is as follows:

$$
w_{n_{1} \hat{1}_{1}, n_{2} i_{2} \gamma_{2}}=\sum_{n i} w_{n_{1} i_{1}, n_{2} i_{2} \gamma_{2}}^{n i},
$$

where

$$
w_{n_{1} i_{1}, n_{2} i_{2} \gamma_{2}}^{n i}=\sum_{\lambda} c_{n i}^{\lambda} w_{n_{1} i_{1}, n_{2} i_{2} \gamma_{2}}^{\lambda n i}, w_{n_{1} i_{1} \gamma_{1}, n_{2} i_{2} \gamma_{2}}^{\lambda n i}=v_{n_{1} i_{1} \gamma_{1}, n_{2} i_{2} \gamma_{2}}^{\lambda n i}+\Delta v_{n_{1} i_{1}, n_{2} i_{2} \gamma_{2}}^{\lambda n i}-v_{n_{1} i_{1}, n_{2} i_{2} \gamma_{2}}^{A n i} \cdot
$$

Here, $c_{n i}^{\lambda}$ are random numbers taking values of 1 or 0 , depending on whether the $\lambda$ kind atom is at the site $(n i)$ or not.

Hamiltonian of electron-phonon interaction (7) is expressed through directional derivative of potential energy in ion-core field along displacement-vector projections of atom, $\mathbf{u}\left(\begin{array}{c}n \\ i\end{array}\right)$. In (7), the value of $v_{n_{1} i_{1}, n_{2} i_{2} \gamma_{2}}^{\prime}$ is given by 


$$
v_{n_{1} i_{1}, n_{2} i_{\gamma_{2}}}^{\prime}=\sum_{n i \alpha} v_{n_{1} \gamma_{1}, n_{2} i_{2} \gamma_{2}}^{\prime n i \alpha} u_{\alpha}\left(\begin{array}{c}
n \\
i
\end{array}\right)
$$

where

$$
v_{n_{1} i_{1}, n_{2} i_{2} \gamma_{2}}^{\prime \prime n i \alpha}=\sum_{\lambda} c_{n i}^{\lambda} v_{n_{1} i_{1} \gamma_{1}, n_{2} i_{2} \gamma_{2}}^{\prime \lambda n i \alpha},
$$

and $v_{n_{1} \dot{i} \gamma_{1}, n_{2} i_{2} \gamma_{2}}^{\lambda n i \alpha}$ is defined by $(11)$, where $v^{\lambda}\left(\mathbf{r}-\mathbf{r}_{n i}\right)$ is replaced with

$$
-e_{n i \alpha} \frac{d}{d\left|\mathbf{r}-\mathbf{r}_{n i}\right|} v^{\lambda}\left(\left|\mathbf{r}-\mathbf{r}_{n i}\right|\right),
$$

where

$$
\mathbf{e}_{n i}=\frac{\mathbf{r}-\mathbf{r}_{n i}}{\left|\mathbf{r}-\mathbf{r}_{n i}\right|} .
$$

Values of $\Delta v_{n_{1} i_{1}, n_{2} i_{2} \gamma_{2}}^{\lambda n i}$ in (11) describe electron scattering on atom static displacements and are defined by

$$
\Delta v_{n_{1} i_{1}, n_{2} i_{2} \gamma_{2}}^{\lambda n i}=\sum_{\alpha} v_{n_{1} i_{1} \gamma_{1}, n_{2} i_{2} \gamma_{2}}^{\prime \lambda i \alpha} u_{\alpha}^{s}\left(\begin{array}{c}
n \\
i
\end{array}\right) .
$$

\section{GREEN'S FUNCTIONS OF ELECTRONS AND PHONONS}

To calculate the energy spectrum of electrons and phonons, free energy and electrical conductivity of disordered crystal, we introduce twotime Green's function. We define two-time retarded $\left(G_{r}^{A B}\left(t, t^{\prime}\right)\right)$ and advanced $\left(G_{a}^{A B}\left(t, t^{\prime}\right)\right)$ Green's functions as follow [30]:

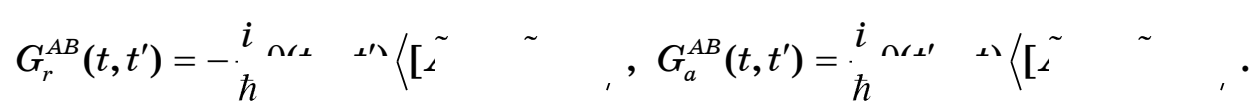

Within the Heisenberg representation, operator

$$
\text { ז } \mathrm{H} / \hbar^{2} \ldots \mathrm{H}^{\hbar} \text {, }
$$

where $\hbar$ is the Planck's constant, $\mathrm{H}=H-\mu_{e}, \mu_{e}$-the chemical potential of electron subsystem, $N_{e}$ - the operator number of electrons:

$$
N_{e}=\sum_{n i \gamma} a_{n i \gamma}^{+} a_{n i \gamma} .
$$

In expression (12), 


$$
[A, B]=A B-\eta B A,
$$

where $\eta=1$ for Bose operators $A, B$, and $\eta=-1$ for Fermi operators $A$, $B ; \theta(t)$ - the Heaviside step function. Brackets $\langle\ldots\rangle$ in (12) denote averaging such as:

$$
\langle A\rangle=\operatorname{Sp}(\rho A), \rho=e^{(\Omega-H) / \Theta},
$$

where $\Omega$ is thermodynamic potential of a system, $\Theta=k_{B} T, T-$ temperature.

Calculation of two-time retarded and advanced Green's functions (12) is based on the calculation of temperature Green functions. Known relation between the spectral representations for retarded, advanced and temperature Green's functions is used.

Let us define the temperature Green's function as

$$
G^{A B}\left(\tau, \tau^{\prime}\right)=-\left\langle T_{\tau^{\prime}} \tilde{\sim} \quad \sim\right.
$$

where operator $\tilde{L}$ is derived from $\tilde{L}$ in (12) by replacing $t=-i \hbar \tau$,

$$
\begin{aligned}
& \sim \quad{ }^{\mathrm{H}} A e^{-\mathrm{H} \tau} ; \\
& T_{\tau^{\perp}} \sim \sim \sim \sim \sim \sim \sim \text {, }
\end{aligned}
$$

$\eta=1$ for Bose operators $A, B$, and $\eta=-1$ for Fermi operators $A, B$.

We introduce the operator

$$
\sigma(\tau)=e^{H_{0} \tau} e^{-H \tau},
$$

where $\mathrm{H}=\mathrm{H}_{0}+H_{\text {int }}, \mathrm{H}_{0}=H_{0}-\mu_{e} N_{e}$. Operator $\sigma(\tau)$ satisfies the equation

$$
\frac{\partial \sigma(\tau)}{\partial \tau}=-H_{\text {int }}(\tau) \sigma(\tau)
$$

where $H_{\text {int }}(\tau)=e^{\mathrm{H}_{0} \tau} H_{\text {int }} e^{-\mathrm{H}_{0} \tau}$.

The solution of Eq. (17) provided that $\sigma(0)=1$ follows from the definition of (16) and has the form

$$
\sigma(\tau)=T_{\tau} \exp \left[-\int_{0}^{\tau} H_{\mathrm{int}}\left(\tau^{\prime}\right) d \tau^{\prime}\right] .
$$

Taking into account expression (16) for the operator within the Heisenberg representation, one can write as follows:

$$
\tilde{i} \quad(\tau) A(\tau) \sigma(\tau) .
$$


Expression (13) for temperature Green's function, in view of (17), leads to equation

$$
G^{A B}\left(\tau, \tau^{\prime}\right)=-\left\langle T_{\tau} A(\tau) B\left(\tau^{\prime}\right) \sigma(1 / \Theta)\right\rangle_{0} /\langle\sigma(1 / \Theta)\rangle_{0},
$$

where

$$
\langle A\rangle_{0}=\operatorname{Sp}\left(\rho_{0} A\right), \rho_{0}=e^{\left(\Omega_{0}-H_{0}\right) / \Theta} .
$$

Expanding the exponent $\sigma(\tau)$ in expression (18) in a series of powers $H_{\text {int }}(\tau)$, substituting the result into (20), and using Wick's theorem for calculating the temperature Green's [28] functions of disordered crystals, it is possible to construct a diagram technique similar to a homogeneous system [29]. The denominator in formulas (20) is derived from the same factor in the numerator. So, Green's functions can be expressed in a series only connected diagrams. Using the relation between the spectral representations of temperature and time Green's functions [29], by analytic continuation to real axis, we obtain the following set of equations for retarded Green functions (hereinafter, index $r$ will be omitted) [28]:

$$
\begin{gathered}
G^{a a^{+}}(\varepsilon)=G_{0}^{a a^{+}}(\varepsilon)+G_{0}^{a a^{+}}(\varepsilon)\left(w+\Sigma_{e f}(\varepsilon)+\Sigma_{e e}(\varepsilon)\right) G^{a a^{+}}(\varepsilon), \\
G^{u u}(\varepsilon)=G_{0}^{u u}(\varepsilon)+G_{0}^{u u}(\varepsilon)\left(\Delta \Phi+\Sigma_{f e}(\varepsilon)+\Sigma_{f f}(\varepsilon)\right) G^{u u}(\varepsilon)+G_{0}^{u P}(\varepsilon) \Delta M^{-1} G^{P u}(\varepsilon), \\
G^{P P}(\varepsilon)=G_{0}^{P P}(\varepsilon)+G_{0}^{P P}(\varepsilon) \Delta M^{-1} G^{P P}(\varepsilon)+G_{0}^{P u}(\varepsilon)\left(\Delta \Phi+\Sigma_{f e}(\varepsilon)+\Sigma_{f f}(\varepsilon)\right) G^{u P}(\varepsilon),(21) \\
G^{u P}(\varepsilon)=G_{0}^{u P}(\varepsilon)+G_{0}^{u P}(\varepsilon) \Delta M^{-1} G^{P P}(\varepsilon)+G_{0}^{u u}(\varepsilon)\left(\Delta \Phi+\Sigma_{f e}(\varepsilon)+\Sigma_{f f}(\varepsilon)\right) G^{u P}(\varepsilon), \\
G^{P u}(\varepsilon)=G_{0}^{P u}(\varepsilon)+G_{0}^{P u}(\varepsilon)\left(\Delta \Phi+\Sigma_{f e}(\varepsilon)+\Sigma_{f f}(\varepsilon)\right) G^{u u}(\varepsilon)+G_{0}^{P P}(\varepsilon) \Delta M^{-1} G^{P u}(\varepsilon),
\end{gathered}
$$

where $\varepsilon=\hbar \omega$.

Here, $G^{a a^{+}}(\varepsilon), G^{u u}(\varepsilon), G^{P P}(\varepsilon), G^{u P}(\varepsilon), G^{P u}(\varepsilon)$ are spectral representation of one-particle Green's function of the electrons' subsystem as well as 'displacement-displacement', 'impulse-impulse', 'displacement-impulse', 'impulse-displacement' Green's functions of phonons' subsystem, respectively; $\Sigma_{e f}(\varepsilon), \Sigma_{f e}(\varepsilon), \Sigma_{e e}(\varepsilon), \Sigma_{f f}(\varepsilon)$ are actual energy parts (mass operators), which describe the electron-phonon, phonon-electron, electron-electron, and phonon-phonon interactions, respectively.

Upon receipt of the expression (21), the spectral decompositions for the two-time (12) and temperature (15) Green's functions are used:

$$
G_{r, a}^{A B}(t)=\frac{1}{2 \pi} \int_{-\infty}^{\infty} G_{r, a}^{A B}(\omega) e^{-i \omega t} d \omega, G_{r, a}^{A B}(\omega)=\int_{-\infty}^{\infty} G_{r, a}^{A B}(t) e^{i \omega t} d t
$$




$$
\begin{gathered}
G^{A B}(\tau)=\Theta \sum_{\omega_{n}} G^{A B}\left(\omega_{n}\right) e^{-i \omega_{n} \tau}, G^{A B}\left(\omega_{n}\right)=\frac{1}{2} \int_{-1 / \Theta}^{1 / \Theta} G^{A B}(\tau) e^{i \omega_{n} \tau} d \tau, \\
\omega_{n}=\left\{\begin{array}{cc}
2 n \pi \Theta & \text { for Bose particles, } \\
(2 n+1) \pi \Theta & \text { for Fermi particles, }
\end{array} \quad n=0, \pm 1, \pm 2, \ldots .\right.
\end{gathered}
$$

There are well-known relations between spectral representations of Green's functions, which follow from Green's functions definitions:

$$
G^{A B}\left(\omega_{n}\right)= \begin{cases}G_{r}^{A B}\left(i \omega_{n} / \hbar\right. & >0, \\ G_{a}^{A B}\left(i \omega_{n} / \hbar\right. & =0 .\end{cases}
$$

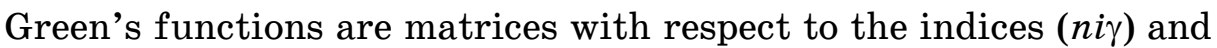
( $n i \alpha)$, respectively, for the subsystem of electrons and phonons.

From the equations of motion for Green's functions of zero approximation [30], one can be obtained:

$$
\begin{aligned}
& G_{0}^{a a^{+}}(\varepsilon)=\left[\varepsilon-H_{0}^{(1)}\right]^{-1}, H_{0}^{(1)}=\left\|h_{n i \gamma, n^{\prime} \gamma^{\prime}}^{(0)}\right\|, G_{0}^{u u}(\varepsilon)=\left[\omega^{2} M_{A}-\Phi^{(0)}\right]^{-1}, \\
& \Phi^{(0)}=\left\|\Phi_{n i \alpha, n^{\prime} i^{\prime} \alpha^{\prime}}^{(0)}\right\|, G_{0}^{P P}(\varepsilon)=\left[\omega^{2}-M_{A}^{-1} \Phi^{(0)}\right]^{-1} \Phi^{(0)},
\end{aligned}
$$

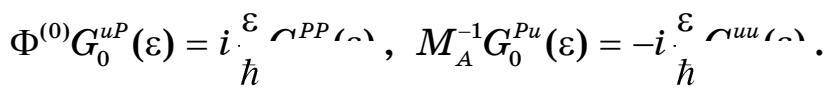

Provided

$$
\left(\begin{array}{lll}
\varepsilon^{2} & \wedge \pi \boldsymbol{\pi} \cdot \Delta \Phi+\Sigma_{f e}(\varepsilon)+\Sigma_{f f}(\varepsilon) \\
\hbar &
\end{array}\right)_{n i \alpha, n^{\prime} i^{\prime} \alpha^{\prime}} / \Phi_{\alpha \alpha^{\prime}}^{(0)}\left(\begin{array}{ll}
n & n^{\prime} \\
i & i^{\prime}
\end{array}\right) \ll<1,
$$

solution of the set (21) has the form:

$$
\begin{gathered}
G^{a a^{+}}(\varepsilon)=\left[\left[G_{0}^{a a^{+}}(\varepsilon)\right]^{-1}-\left(w+\Sigma_{e f}(\varepsilon)+\Sigma_{e e}(\varepsilon)\right)\right]^{-1}, \\
G^{u u}(\varepsilon)=\left[\left[G_{0}^{u u}(\varepsilon)\right]^{-1}-\left(\begin{array}{l}
\left.\left.\varepsilon^{2} \wedge \pi r \cdot \Delta \Phi+\Sigma_{f e}(\varepsilon)+\Sigma_{f f}(\varepsilon)\right)\right]^{-1}, \\
\hbar
\end{array}\right.\right. \\
\Delta M^{-1} G^{P P}(\varepsilon)={ }_{\varepsilon^{2} \wedge \wedge \pi \sim u u}(\varepsilon),
\end{gathered}
$$

where $\Delta M=\left\|\left(M_{A}-M_{n i}\right) \delta_{n n^{\prime}} \delta_{i i^{\prime}} \delta_{\alpha \alpha^{\prime}}\right\|$.

Upon receipt of Eqs (24), the members, which are proportional to the second and higher powers of small parameter (23), are neglected.

Using the mentioned above diagram technique, in work [28], explicit 


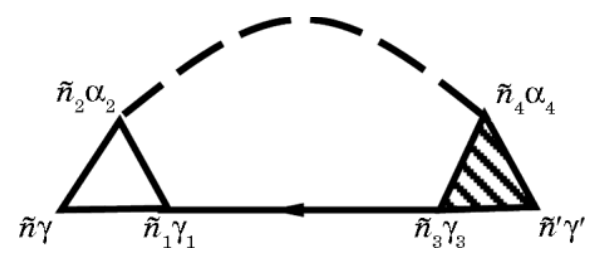

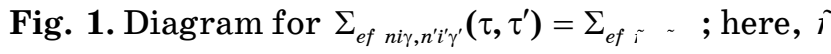

expression has been found for the mass operator of Green's functions that describe the many-particle interactions in the system.

The mass operator of Green's function of electrons for the electronphonon interaction, $\Sigma_{e f}\left(\tau, \tau^{\prime}\right)$, is described by the diagram in Fig. 1. Solid lines in the Fig. 1 correspond to the Green's function of electrons, $G_{n i \gamma, n^{\prime} i^{\prime} \gamma^{\prime}}^{a a^{+}}\left(\tau, \tau^{\prime}\right)$; dashed lines correspond to the Green's function of phonons, $G_{n i \alpha, n^{\prime} i^{\prime} \alpha^{\prime}}^{u u}\left(\tau, \tau^{\prime}\right)$. Vertex part $\Gamma_{n i \gamma, n_{1} \gamma_{1} \gamma_{1}}^{n_{2} i_{2} \alpha_{2}}\left(\tau_{2}, \tau, \tau_{1}\right)$ is described by diagrams in Fig. 2, where unshaded triangle corresponds to the ex-

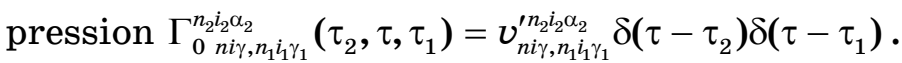

For internal points $\tilde{i}$ in Figs. 1 and 2, summation is carried out. Summation by $\tilde{i}$ provides summation by ni $i$ and integration over $\tau$. Expressions corresponding each diagram attribute multiplier $(-1)^{n+F}$, where $n$ is order of diagram (number of vertices $\Gamma_{0}$ in the diagram), and $F$ is the number of lines for the Green's function of electrons, $G^{a a^{+}}$, which goes out and goes into in the same vertices.

For the mass operator that describes electron-phonon interaction, we have:

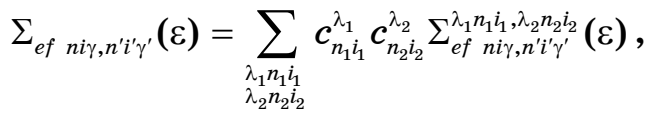

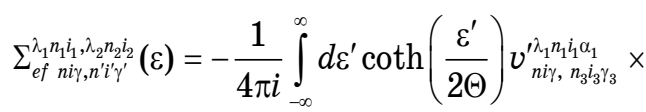

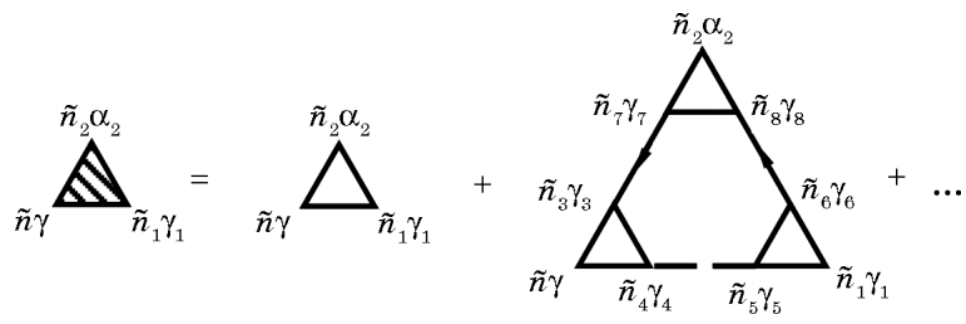

Fig. 2. Diagrams for the vertex part $\Gamma_{n i \gamma, n_{1} i_{\gamma} \gamma_{1}}^{n_{2} i_{2} \alpha_{2}}\left(\tau_{2}, \tau, \tau_{1}\right)=\Gamma_{\tilde{r}}^{\tilde{r}} \quad ;$ here, $\tilde{\tau}$ 


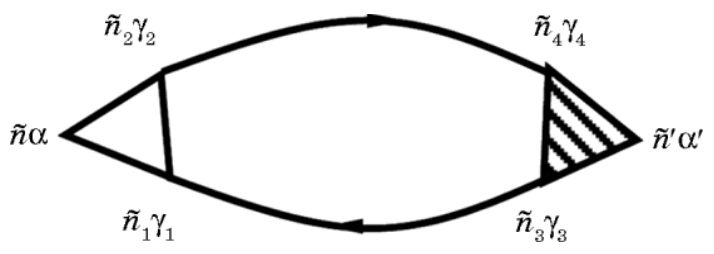

Fig. 3. Diagram for $\Sigma_{f e n i \alpha, n^{\prime} i^{\prime} \alpha^{\prime}}\left(\tau, \tau^{\prime}\right)=\Sigma_{f e} \tau \quad$; here, $\tilde{\tau}$

$$
\times\left[G_{n_{1} i_{1} \alpha_{1}, n_{2} i_{2} \alpha_{2}}^{u u}\left(\varepsilon^{\prime}\right)-G_{n_{2} i_{2} \alpha_{2}, n_{1} i_{1} \alpha_{1}}^{u u^{*}}\left(\varepsilon^{\prime}\right)\right] G_{n_{3} i_{3} \gamma_{3}, n_{4} i_{4} \gamma_{4}}^{a a^{+}}\left(\varepsilon-\varepsilon^{\prime}\right) \Gamma_{n_{4} i_{4} \gamma_{4}, n^{\prime} i^{\prime} \gamma^{\prime}}^{\lambda_{2} n_{2} i_{2} \alpha_{2}}\left(\varepsilon-\varepsilon^{\prime}, \varepsilon ; \varepsilon^{\prime}\right) .
$$

For repeated indices, summation is performed.

The diagram in Fig. 3 describes the phonon-electron interaction. Designations in Fig. 3 correspond to designations in Figs. 1 and 2.

The mass operator describing phonon-electron interaction has the form:

$$
\begin{aligned}
& \Sigma_{f e n i \alpha, n^{\prime} i^{\prime} \alpha^{\prime}}(\varepsilon)=\sum_{\lambda \lambda^{\prime}} c_{n i}^{\lambda} c_{n^{\prime} i^{\prime}}^{\lambda^{\prime}} \Sigma_{f e n i \alpha, n^{\prime} i^{\prime} \alpha^{\prime}}^{\lambda \lambda^{\prime}}(\varepsilon), \\
& \sum_{f e n i \alpha, n^{\prime} i^{\prime} \alpha^{\prime}}^{\lambda \lambda^{\prime}}(\varepsilon)=\frac{1}{2 \pi i} \int_{-\infty}^{\infty} d \varepsilon^{\prime} f\left(\varepsilon^{\prime}\right) v_{n_{2} i_{2} \gamma_{2}, n_{1} i_{1} \gamma_{1}}^{\prime \lambda \lambda i \alpha} \times \\
& \times\left\{\left[G_{n_{1} a_{1} \gamma_{1}, n_{3} i_{3} \gamma_{3}}^{a{ }^{+}}\left(\varepsilon+\varepsilon^{\prime}\right)-G_{n_{3} i_{3} \gamma_{3}, n_{1} i_{\gamma_{1}}}^{a a^{+}}\left(\varepsilon+\varepsilon^{\prime}\right)\right] G_{n_{2} i_{2} \gamma_{2}, n_{4} i_{4} \gamma_{4}}^{a a^{+} *}\left(\varepsilon^{\prime}\right)+\right.
\end{aligned}
$$

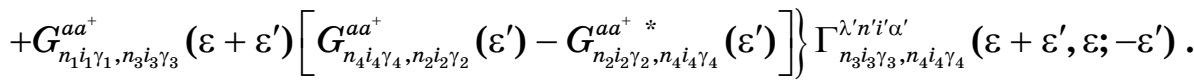

Diagrams for the mass operator $\Sigma_{e e}\left(\tau, \tau^{\prime}\right)$ that describes electronelectron interaction are shown in Fig. 4.

Vertex part $\Gamma_{n i \gamma, n^{\prime} i^{\prime} \gamma^{\prime}}^{n_{2} i_{\gamma} n_{1} n_{1} \gamma_{1}}\left(\tau_{2}, \tau_{1}, \tau, \tau^{\prime}\right)$ is shown by diagrams in Fig. 5. Unshaded triangle in Fig. 5 corresponds to the expression

$$
\begin{aligned}
& \Gamma_{0 \text { niv } n^{\prime}, n^{\prime} \gamma^{\prime} \gamma^{\prime}}^{n_{2} i_{2} \gamma_{2}, n_{1} i_{1}}\left(\tau_{2}, \tau_{1}, \tau, \tau^{\prime}\right)=\tilde{i} \quad j\left(\tau-\tau_{2}\right) \delta\left(\tau-\tau_{1}\right) \delta\left(\tau-\tau^{\prime}\right),
\end{aligned}
$$

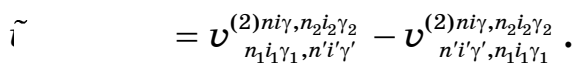

$$
\begin{aligned}
& \sum_{\tilde{n} \gamma}^{\tilde{n}_{2} \gamma_{2}}+\tilde{n} \gamma
\end{aligned}
$$

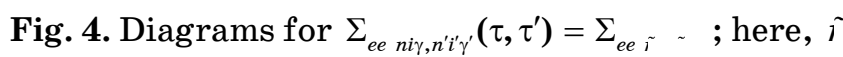




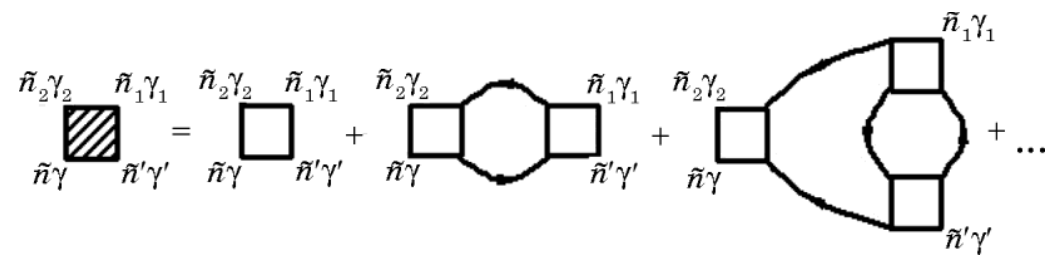

Fig. 5. Diagrams for vertex part $\Gamma_{n i \gamma, n^{\prime} i^{\prime} \gamma^{\prime}}^{n_{i} i_{1} \gamma_{1}, i_{1} \gamma_{1}}\left(\tau_{2}, \tau_{1}, \tau, \tau^{\prime}\right)=\Gamma_{\tilde{i}}^{\tilde{i}} \sim \quad$; here, $\tilde{i}$

The mass operator that describes electron-electron interaction is as follows:

$$
\begin{aligned}
& \sum_{e e n i \gamma, n^{\prime} i^{\prime} \gamma^{\prime}}(\varepsilon)=\sum_{e e ~ n i \gamma, n^{\prime} \gamma^{\prime} \gamma^{\prime}}^{(1)}+\sum_{e e}^{(2)} n i \gamma, n^{\prime} \gamma^{\prime} \gamma^{\prime}(\varepsilon),
\end{aligned}
$$

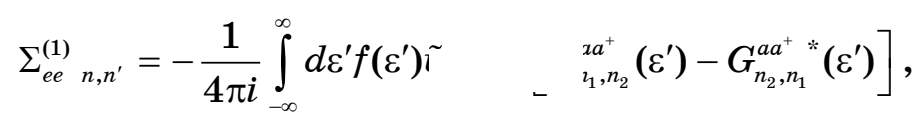

$$
\begin{aligned}
& \Sigma_{e e n, n^{\prime}}^{(2)}(\varepsilon)=-\left(\frac{1}{2 \pi i}\right)^{2} \int_{-\infty}^{\infty} d \varepsilon_{1} \int_{-\infty}^{\infty} d \varepsilon_{2} \tilde{\imath} \\
& \times\left\{f\left(\varepsilon_{1}\right) f\left(\varepsilon_{2}\right)\left[G_{n_{5}, n_{2}}^{a a^{+} *}\left(\varepsilon-\varepsilon_{1}+\varepsilon_{2}\right) G_{n_{1}, n_{4}}^{a a^{+}}\left(\varepsilon_{1}\right)-G_{n_{2}, n_{5}}^{a a^{+}}\left(\varepsilon-\varepsilon_{1}+\varepsilon_{2}\right) G_{n_{4}, n_{1}}^{a a^{+} *}\left(\varepsilon_{1}\right)\right] \times\right. \\
& \times\left[G_{n_{6}, n_{3}}^{a a^{+}}\left(\varepsilon_{2}\right)-G_{n_{3}, n_{6}}^{a a^{+} *}\left(\varepsilon_{2}\right)\right] \Gamma_{n_{4}, n^{\prime}}^{n_{5}, n_{6}}\left(\varepsilon_{1}, \varepsilon-\varepsilon_{1}+\varepsilon_{2} ; \varepsilon_{2}, \varepsilon\right)+ \\
& +f\left(\varepsilon_{1}\right) f\left(\varepsilon_{1}+\varepsilon_{2}-\varepsilon\right)\left[G_{n_{1}, n_{4}}^{a a^{+}}\left(\varepsilon_{1}\right) G_{n_{6}, n_{3}}^{a a^{+}}\left(\varepsilon_{1}+\varepsilon_{2}-\varepsilon\right)-G_{n_{4}, n_{1}}^{a a^{+*}}\left(\varepsilon_{1}\right) G_{n_{3}, n_{6}}^{a a^{+*}}\left(\varepsilon_{1}+\varepsilon_{2}-\varepsilon\right)\right] \times \\
& \left.\times\left[G_{n_{2}, n_{5}}^{a a^{+}}\left(\varepsilon_{2}\right)-G_{n_{5}, n_{2}}^{a a^{+} *}\left(\varepsilon_{2}\right)\right] \Gamma_{n_{4}, n^{\prime}}^{n_{5}, n_{6}}\left(\varepsilon_{1}, \varepsilon_{2} ; \varepsilon_{1}+\varepsilon_{2}-\varepsilon, \varepsilon\right)\right\}, \\
& \tilde{i} \quad \underset{n_{1}, n^{\prime}}{(2) n, n_{2}}-v_{n^{\prime}, n_{1}}^{(2) n, n_{2}}(n \equiv n i \gamma) \text {. }
\end{aligned}
$$

Similarly, in Ref. [28], expressions are obtained for the mass operator $\Sigma_{f f}(\varepsilon)$ that describes phonon-phonon interactions.

Upon receipt of expressions (25)-(27), the relations arising from the theory of functions of complex variables are used:

$$
\begin{array}{cc}
\Theta \sum_{\omega_{n}} \varphi\left(i \omega_{n}\right)=\frac{1}{4 \pi i} \bigcirc & ) \varphi(z)\left(\omega_{n}=2 n \pi \Theta\right), \\
\Theta \sum_{\omega_{n}} \varphi\left(i \omega_{n}\right)=-\frac{1}{2 \pi i} \bigcirc & \left(\omega_{n}=(2 n+1) \pi \Theta\right), \\
f\left(\frac{z}{\Theta}\right)=\left[\exp \left(\frac{z}{\Theta}\right)+1\right]^{-1},
\end{array}
$$


where $\varphi(z)$ is the analytical function of complex $z$ in the region covered by a contour $C$.

Between the Green's functions (12) $G_{\mu}(\varepsilon)$ defined at a constant chemical potential $\mu_{e}$ and the Green's function $G_{N}(\varepsilon)$ defined at a constant number of electrons $N_{e}$, there exists a relation $G_{\mu}(\varepsilon)=G_{N}(\varepsilon+\mu)$.

When renormalization of vertex parts is neglected, diagrams for the mass operators in expressions (25)-(27) should be put as follow:

$$
\Gamma_{n_{4} i_{4} \gamma_{4}, n^{\prime} \gamma^{\prime}}^{\lambda_{2} n_{2} i_{2} \alpha_{2}}\left(\varepsilon-\varepsilon^{\prime}, \varepsilon ; \varepsilon^{\prime}\right)=v_{n_{4}^{4} l_{4}, n^{\prime} i^{\prime} \gamma^{\prime}}^{\prime \lambda_{2} n_{2} i_{2} \alpha_{2}}, \Gamma_{n_{4}, n^{\prime}}^{n_{5}, n_{6}}\left(\varepsilon_{1}, \varepsilon-\varepsilon_{1}+\varepsilon_{2} ; \varepsilon_{2}, \varepsilon\right)=\tilde{r} \quad .
$$

In expressions (26), (27), $f(\varepsilon)$ is the Fermi function; Fermi level $\varepsilon_{F} \equiv \mu_{e}$ of a system is determined by the equation:

$$
\langle Z\rangle=\int_{-\infty}^{\infty} f\left(\varepsilon, \varepsilon_{F}\right) g_{e}(\varepsilon) d \varepsilon,
$$

where $\langle Z\rangle$ - the average number of electrons per atom. Electron density of states, $g_{e}(\varepsilon)$, in expression (28) is given by the formula:

$$
g_{e}(\varepsilon)=\frac{1}{v} \sum_{i, \gamma, \sigma, \lambda} P_{n i}^{\lambda} g_{n i \gamma \sigma}^{\lambda}(\varepsilon),
$$

where $P_{n i}^{\lambda}=\left\langle c_{n i}^{\lambda}\right\rangle$ - the probability of filling the site $(n i)$ with the $\lambda$ kind atom, $v$ - the number of atoms in a primitive cell, $g_{n i \gamma \sigma}^{\lambda}(\varepsilon)$ - the conditional partial electron density of states per one atom for state $(n i \gamma \sigma)$ provided the $\lambda$ kind atom be placed at the site $(n i)$ :

$$
g_{n i \gamma \sigma}^{\lambda}(\varepsilon)=-\left.\frac{1}{\pi} \operatorname{Im}\left\langle G_{n i \gamma \sigma, n i \gamma \sigma}^{a a^{+}}(\varepsilon)\right\rangle\right|_{\lambda \in(n i)} .
$$

In the last formula, configuration averaging is carried out provided that, in site $(n i)$, the $\lambda$ kind atom is placed.

Equation (28) follows from the definitions of electrons' number operator (12) and Green's function $G^{A B}\left(\tau, \tau^{\prime}\right)(15) ; A=a_{n i \gamma}^{+}, B=a_{n i \gamma}$ [27].

It should be noted that the first term $\Sigma_{e e}^{(1)}{ }_{n i \gamma, n^{\prime} \gamma^{\prime} \gamma^{\prime}}$ in the expression for the mass operator $\Sigma_{e e n i \gamma, n^{\prime} i^{\prime} \gamma^{\prime}}(\varepsilon)$ of electron-electron interactions is described the Coulomb and exchange electron-electron interactions within the Hartree-Fock approximation. The second term $\Sigma_{e e n i \gamma, n^{\prime} i^{\prime} \gamma^{\prime}}^{(2)}(\varepsilon)$, which is caused by output beyond the Hartree-Fock approximation, describes the electron correlations.

Expressions (24) differ from the corresponding expressions for the Green's function of the single-particle Hamiltonians of disordered system only by the view of mass operators. Therefore, to calculate the 
Green's function (24), the well-known methods of the theory of disordered systems [28] will be used.

Let us perform cluster expansion for Green's functions $G^{a a^{+}}(\varepsilon)$, $G^{u u}(\varepsilon)$ in expressions (24) by introducing the mass operator as the sum of one-site operators and selecting a zero one-site approximation of the Green's function of an effective environment. Specified expansion is a generalization of the cluster expansion for the Green's function $G^{a a^{+}}(\varepsilon)$ of single-particle Hamiltonian. Green's function of an effective environment for the subsystem of electrons determine the expression [28]

$$
\text { i } \left.\quad\left[G_{0}^{a a^{+}}(\varepsilon)\right]^{-1}-\left(\tilde{:}^{\sim} \quad \varepsilon\right)\right]^{-1} \text {, }
$$

where the mass operator of the electron-phonon interaction for the $A$ kind atoms in the effective environment is equal to

The values : $\quad \varepsilon$ ) are determined by expression (25), in which the Green's functions of disordered crystal are replaced by Green's functions of the effective environment.

Green's functions of an effective environment for phonon subsystem are defined by expression:

$$
\text { ( } \left.\left.\quad\left[G_{0}^{u u}(\varepsilon)\right]^{-1}-(\tilde{:} \quad \sim \quad \varepsilon)\right)\right]^{-1} .
$$

The values of $: \quad, \tilde{:} \quad, \tilde{:} \quad$ in expressions (30) and (31) are defined similarly to :

In expressions (30) and (31), $\sigma_{e}(\varepsilon), \sigma_{f}(\varepsilon)$ are potentials of an effective environment (coherent potentials); their values will be determined.

Green's functions (24) satisfy the equation:

$$
G(\varepsilon)=\tilde{i} \sim \sim \sim
$$

where $T$ is a matrix of scattering, which can be represented as a series with terms describing the scattering by clusters with different numbers of sites:

$$
T=\sum_{\left(n_{1} i_{1}\right)} t^{n_{1} i_{1}}+\sum_{\left(n_{1} i_{1}\right) \neq\left(n_{2} i_{2}\right)} T^{(2) n_{1} i_{1}, n_{2} i_{2}}+\ldots
$$

Here, $T^{(2) n_{1} i_{1}, n_{2} i_{2}}=\left[I-t^{n_{1} \dot{i}_{1}} \tilde{C}\right.$ , and $t^{n_{1} i_{1}}$ is operator of scattering on the same site that is determined by the expression:

$$
t^{n_{1} i_{1}}=\left[I-\left(\Sigma^{n_{1} \dot{1}_{1}}-\sigma^{n_{1} \dot{i}_{1}}\right) \tilde{c}, \quad-\sigma^{n_{1} \dot{1}_{1}}\right) .
$$


Value $\Sigma_{e}^{n_{1} i_{1}}(\varepsilon)$ in the formula (39) for the subsystem of electrons is given by the expression

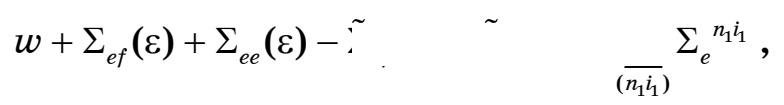

and, for the subsystem of phonons, by the expression

$$
\varepsilon^{2} \wedge \boldsymbol{\pi} \cdot \Delta \Phi+\Sigma_{f e}(\varepsilon)+\Sigma_{f f}(\varepsilon)-\tilde{\imath} \sim \quad \sim \quad \sum_{\left(n_{1} i_{1}\right)} \Sigma_{f}^{n_{1} i_{1}} .
$$

Coherent potentials determined from the condition $\left\langle t^{0 i_{1}}\right\rangle=0$ satisfy the set of coupled equations [28]:

$$
\begin{gathered}
\sigma_{e}^{0 i_{1}}(\varepsilon)=\left\langle\left[1-\left(\Sigma_{e}^{0 i_{1}}(\varepsilon)-\sigma_{e}^{0 i_{1}}(\varepsilon)\right) \tilde{c} \quad\right\rangle^{-1} \times\right. \\
\times\left\langle\left[1-\left(\Sigma_{e}^{0 i_{1}}(\varepsilon)-\sigma_{e}^{0 i_{1}}(\varepsilon)\right) \tilde{c}\right\lrcorner \Sigma_{e}^{0 i_{1}}(\varepsilon)\right\rangle,
\end{gathered}
$$

and, for the subsystem of phonons,

$$
\begin{gathered}
\sigma_{f}^{0 i_{1}}(\varepsilon)=\left\langle\left[ 1-\left(\Sigma_{f}^{0 i_{1}}(\varepsilon)-\sigma_{f}^{0 i_{1}}(\varepsilon)\right) \tilde{c} \quad{ }^{-1} \times\right.\right. \\
\quad \times\left\langle\left[1-\left(\Sigma_{f}^{0 i_{1}}(\varepsilon)-\sigma_{f}^{0 i_{1}}(\varepsilon)\right) \tilde{c} \quad \Sigma_{f}^{0 i_{1}}(\varepsilon)\right\rangle .\right.
\end{gathered}
$$

Using (33), cluster decomposition for the Green's function of electrons and phonons of disordered crystal can be obtained. As a zero onesite approximation in this method of cluster expansion, the approximation of coherent potential is chosen. The densities of electrons' and phonons' states, free energy and electrical conductivity are presented as infinite series with terms describing the processes of scattering on clusters with different numbers of atoms. As shown, the contributions of scattering processes with electrons and phonons in clusters decrease with increasing number of atoms in the cluster by a small parameter:

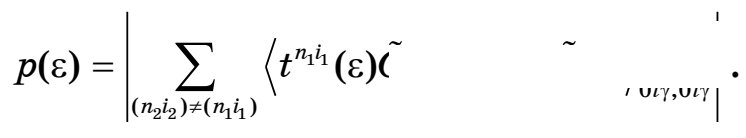

Investigation of this parameter shows that it is small in a wide range of changes of characteristics of the system (including the concentration of components), except for narrow intervals of energy values at the edges of the spectrum. For single-electron Hamiltonian in single-band model, this parameter was introduced for the first time and investi- 
gated in Ref. [31]. Subsequently, this parameter was investigated in Ref. [32]. For many-particle Hamiltonian, which includes electronelectron and electron-phonon interactions, this parameter was explored in Ref. [28].

Using (29), (32), (33), spending averaging over the distribution of atoms of different kinds at the sites of a crystal lattice, and neglecting the contribution of processes of electron scattering in clusters consisting of three or more atoms, which are small by the above parameter, for the density of electron states, we obtain

$$
g_{e}(\varepsilon)=\frac{1}{v} \sum_{i, \gamma, \sigma, \lambda} P_{0 i}^{\lambda} g_{0 i \gamma \sigma}^{\lambda}(\varepsilon)
$$

$$
\begin{aligned}
& g_{0 i \gamma \sigma}^{\lambda}(\varepsilon)= \\
& =-\frac{1}{\pi} \operatorname{Im}\{\tilde{c}
\end{aligned}
$$

$$
\begin{gathered}
\underset{\substack{(l j) \neq(0 i) \\
\lambda^{\prime}}}{T^{(2) \lambda 0 i, \lambda^{\prime} l j}=\left[I-t^{\lambda} 0 i \tilde{C}\right.}
\end{gathered}
$$

Doing the above-configuration averaging the Green's function $G^{u u}(\varepsilon)$ for the phonon density of states similarly, we obtain

$$
g_{f}(\varepsilon)=\frac{1}{v} \sum_{i, \alpha, \lambda} P_{0 i}^{\lambda} g_{0 i \alpha}^{\lambda}(\varepsilon),
$$

$g_{0 i \alpha}^{\lambda}(\varepsilon)=$

$=-\frac{2 \varepsilon M_{A}}{\pi} \hbar$ T r

where $\tilde{C}$

Matrix elements of the Green's function of the electron subsystem of the effective environment can be calculated, using the Fourier transformation:

$$
\tilde{(} \quad \varepsilon)=\frac{1}{N} \sum_{\mathbf{k}}\left[\varepsilon-H^{(1)}(\mathbf{k})\right]_{i \gamma \sigma,,^{\prime} \gamma^{\prime} \sigma^{\prime}}^{-1} e^{i \mathbf{k} \cdot\left(\mathbf{r}_{n}+\boldsymbol{\rho}_{i}-r_{n^{\prime}}-\boldsymbol{\rho}_{i}\right)},
$$

where

$$
H^{(1)}(\mathbf{k})=H_{0}^{(1)}(\mathbf{k})+\tilde{\imath}^{\sim} \quad \sim \quad(\mathbf{k}, \varepsilon),
$$

$N$-the total number of primitive cells. 
Calculation of matrix elements of Green's functions of phonons' subsystem of an effective environment is performed by the formula

$$
\tilde{\mathbf{c}} \quad \quad \quad=\frac{1}{N} \sum_{\mathbf{k}}\left[\omega^{2} M_{A}-\Phi(\mathbf{k})\right]_{i \alpha, i^{\prime} \alpha^{\prime}}^{-1} e^{i \mathbf{k} \cdot\left(\mathbf{r}_{n}+\boldsymbol{p}_{i}-\mathbf{r}_{n^{\prime}}-\boldsymbol{p}_{i}^{\prime}\right)},
$$

where

$$
\Phi(\mathbf{k})=\Phi^{(0)}(\mathbf{k})+\tilde{\Sigma^{\prime}} \quad \sim \quad(\mathbf{k}, \varepsilon) .
$$

In formulas (41)-(44), vector $\mathbf{k}$ is varying within the first Brillouin zone.

Components of matrix of force constants, which are caused by the direct Coulomb interaction of ions, have the form:

$$
\begin{gathered}
\Phi_{\alpha \alpha^{\prime}}\left(\begin{array}{cc}
n & n^{\prime} \\
i & i^{\prime}
\end{array}\right)=-\frac{Z_{n i} Z_{n i^{\prime}} e^{2}}{4 \pi \varepsilon_{0}\left|\mathbf{r}_{n}+\rho_{i}-\mathbf{r}_{n^{\prime}}-\rho_{i^{\prime}}\right|^{5}} \times \\
\times\left[3\left(r_{n \alpha}+\rho_{i \alpha}-r_{n^{\prime} \alpha}-\rho_{i^{\prime} \alpha}\right)\left(r_{n \alpha^{\prime}}+\rho_{i \alpha^{\prime}}-r_{n^{\prime} \alpha^{\prime}}-\rho_{i^{\prime} \alpha^{\prime}}\right)-\left|\mathbf{r}_{n}+\rho_{i}-\mathbf{r}_{n^{\prime}}-\rho_{i^{\prime}}\right|^{2} \delta_{\alpha \alpha^{\prime}}\right],
\end{gathered}
$$

where $Z_{n i}$-the valence of ion, which is located at the site $(n i) \neq\left(n^{\prime} i^{\prime}\right)$.

Diagonal (by number (ni) of a site) elements of the matrix of force constant are determined from the condition

$$
\sum_{n^{\prime} i^{\prime}} \Phi_{\alpha \alpha^{\prime}}\left(\begin{array}{cc}
n & n^{\prime} \\
i & i^{\prime}
\end{array}\right)=0 .
$$

Value of $\Phi^{(0)}(\mathbf{k})$ in expression (44) is the Fourier image of matrix, $\Phi_{\alpha \alpha^{\prime}}^{(0)}\left(\begin{array}{cc}n & n^{\prime} \\ i & i^{\prime}\end{array}\right)$, given by expression (45); here, $Z_{n i}=Z_{n^{\prime} i^{\prime}}=Z^{A}$, where $Z^{A}$ is the $A$ kind ion valence.

The mass operator : tions of ions by means of electrons.

Single-centre scattering operator $t^{\lambda n_{1} i_{1}}$ in the formula (39) is given by expression (33), in which the quantity $\Sigma_{e}^{\lambda n_{1} i_{1}}(\varepsilon)$ describes the scattering of electrons, according to (11), (25), (27), (34), and has the form:

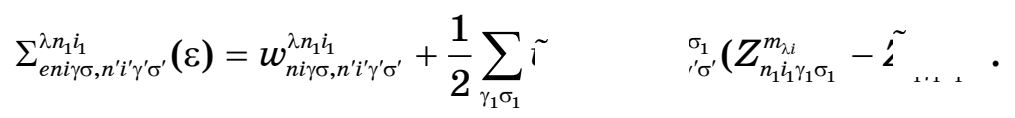

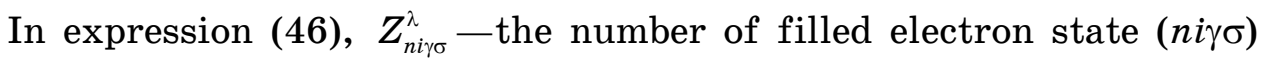
provided that, at the site ( $n i)$, the $\lambda$ kind atom is located. The value $Z_{n i \gamma \sigma}^{\lambda}$ is determined by the expression (28), in which the electron den- 
sity of states, $g_{e}(\varepsilon)$, is replaced by conventional partial density of states, $g_{n i \gamma \sigma}^{\lambda}(\varepsilon)$. The value $Z_{n i \gamma \sigma}^{\lambda}$ is equal to

$$
Z_{n i \gamma \sigma}^{\lambda}=Z_{i \gamma \sigma}^{\lambda}=\int_{-\infty}^{\infty} f\left(\varepsilon, \varepsilon_{F}\right) g_{n i \gamma \sigma}^{\lambda}(\varepsilon) d \varepsilon .
$$

The total number of electrons in the valent states of the $\lambda$ kind atom is equal to

$$
Z_{\lambda i}=\sum_{\gamma \sigma} Z_{i \gamma \sigma}^{\lambda} \cdot
$$

The effective charge of the $\lambda$ kind atom is determined by the expression

$$
\Delta Z_{\lambda i}=Z_{\lambda i}-Z_{\lambda}^{0}
$$

Here, $Z_{\lambda}^{0}$ is the number of electrons in the valent states of noninteracting $\lambda$ kind atoms.

Localized magnetic moment of the $\lambda$ kind atom is equal to

$$
m_{\lambda i}=\sum_{\gamma}\left(Z_{i \gamma \sigma}^{\lambda}-Z_{i \gamma-\sigma}^{\lambda}\right) .
$$

The value $\tilde{i}$ in the formula (47) is derived from expressions (39) and (47) by replacing of the Green's function to the function of effective environment.

Single-centre operator of scattering $t^{\lambda n_{1} i_{1}}$ in the formula (40) is given by expression (33), in which the quantity $\Sigma_{f}^{\lambda n_{1} i_{1}}(\varepsilon)$ that describes, according to (24), (26), (35), the scattering of phonons, has the form

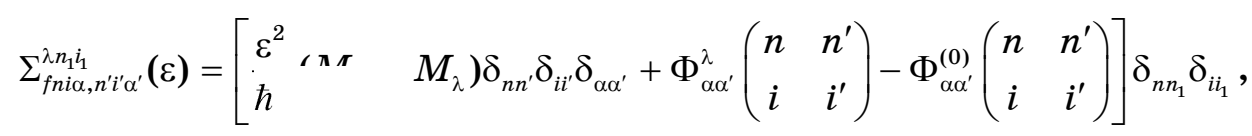

where $\Phi_{\alpha \alpha^{\prime}}^{\lambda}\left(\begin{array}{cc}n & n^{\prime} \\ i & i^{\prime}\end{array}\right)$ is determined by expressions (45) and (44), in which $Z_{n i}=Z^{\lambda}, Z_{n^{\prime i^{\prime}}}=Z^{A}$.

The value $\tilde{Z^{\prime}} \sim \quad \sim \quad$ in expression (42) and second component in right part of expression (46) describes the Coulomb interaction of electrons, including long-range Coulomb interaction at different sites in crystal lattice. The value : in expression (42) and second component in right part of expression (46) describes the exchange interactions of electrons. The value $\tilde{\text { in }}$ in expression (42) describes the electron correlations. Unlike the Refs [11-17], in our 
work, long-range Coulomb interaction of electrons at different sites in crystal lattice is described with considering of arbitrary numbers of energy bands.

The value : in expression (42) describes the scattering of electrons by crystal-lattice vibrations.

In expressions (39), (40), $P_{l j 0 i}^{\lambda^{\prime} / \lambda}$ is conditional probability to find at the site $(l j)$ the $\lambda^{\prime}$ kind atom provided that, at the site $(0 i)$, the $\lambda$ kind atom. $t_{n i}^{\lambda}$ is the value of matrix elements of single-centre operators of scattering for the case when, at the site ( $n i)$, the $\lambda$ kind atom is located.

Probability of filling of site ( $0 i$ ) by the $\lambda$ kind atom in expression (39) is equal:

$$
P_{i 0}^{\lambda}=\left\langle c_{i 0}^{\lambda}\right\rangle,
$$

where $c_{n i}^{\lambda}$ is the random numbers taking values of 1 or 0 depending on the $\lambda$ kind atom located at the site $(n i)$ or not.

For crystals with two kinds of atoms and two sublattice types, $P_{0 i}^{A}=x_{A}-\frac{v_{2}}{v} \eta_{a}$ for $v_{1}$ sublattices of first type and $P_{0 i}^{A}=x_{A}+\frac{v_{1}}{v} \eta_{a}$ for $v_{2}$ sublattices of second type; $P_{0 i}^{B}=1-P_{0 i}^{A} ; v=v_{1}+v_{2} ; x_{A}, x_{B}=1-x_{A}-$ concentrations of components $A, B$ in alloy, respectively; $\eta_{a}$-the parameter of long-range atomic order.

Conditional probability $P_{l j}^{\lambda^{\prime} / \lambda}$ in (39) is defined by the expression

$$
P_{l j 0 i}^{\lambda \lambda \lambda}=P_{0 i}^{\lambda} P_{l j 0 i}^{\lambda^{\prime} / \lambda}=\left\langle c_{l j}^{\lambda^{\prime}} c_{0 i}^{\lambda}\right\rangle
$$

and is related to parameters of interatomic pair correlation $\varepsilon_{l j 0 i}^{B B}$ by next equation $[25,29]$ :

$$
P_{l j 0 i}^{\lambda^{\prime} / \lambda}=P_{l j}^{\lambda^{\prime}}+\frac{\varepsilon_{l j 0 i}^{B B}}{P_{0 i}^{\lambda}}\left(\delta_{\lambda^{\prime} B}-\delta_{\lambda^{\prime} A}\right)\left(\delta_{\lambda B}-\delta_{\lambda A}\right)
$$

here, $\delta$-the Kronecker symbols; $\varepsilon_{l j 0 i}^{B B}=\left\langle\left(c_{l j}^{B}-c_{j}^{B}\right)\left(c_{0 i}^{B}-c_{i}^{B}\right)\right\rangle$.

\section{FREE ENERGY}

Thermodynamic potential of the system is defined by the expression [29]

$$
\Omega=-\Theta \ln \operatorname{Sp}\left(e^{-H / \Theta}\right) .
$$

Hamiltonian $H$ is defined in expression (12). Using the formula (14), 
from (55), we obtain

$$
\Omega=\Omega_{0}+\Omega^{\prime}, \Omega^{\prime}=-\Theta \ln \langle\sigma(1 / \Theta)\rangle_{0},
$$

where $\Omega_{0}$ - the thermodynamic potential of noninteracting electrons and phonons.

For the thermodynamic potential $\Omega^{\prime}(56)$, diagram technique can be developed, which is similar to the diagram technique for the temperature Green's function.

As a result, we obtain

$$
\begin{gathered}
\Omega^{\prime}=-\frac{1}{\pi v N} \operatorname{Im} \int_{0}^{1} \frac{d \lambda}{\lambda} \int_{-\infty}^{\infty} d \varepsilon\left[f(\varepsilon) S p\left\langle\left(w(\lambda)+\Sigma_{e f}(\varepsilon, \lambda)+\Sigma_{e e}(\varepsilon, \lambda)\right) G^{a a^{+}}(\varepsilon, \lambda)\right\rangle+\right. \\
\left.+\frac{1}{2} \operatorname{coth}\left(\frac{\varepsilon}{2 \Theta}\right) \operatorname{Sp}\left\langle\Delta M^{-1}(\lambda) G^{P P}(\varepsilon, \lambda)+\left(\Delta \Phi(\lambda)+\Sigma_{f f}(\varepsilon, \lambda)\right) G^{u u}(\varepsilon, \lambda)\right\rangle\right] .
\end{gathered}
$$

The thermodynamic potential $\Omega_{0}$ in the absence of interaction in the formula (56) is equal to

$$
\Omega_{0}=\Omega_{c}+\Omega_{0 e}+\Omega_{0 f} .
$$

Thermodynamic potential of the electron subsystem is as follows:

$$
\Omega_{0 e}=-\Theta \int_{-\infty}^{\infty} \ln \left(1+e^{\left(\mu_{e}-\varepsilon\right) / \Theta}\right) g_{0 e}(\varepsilon) d \varepsilon .
$$

Thermodynamic potential of the phonon subsystem is

$$
\Omega_{0 f}=\Theta \int_{-\infty}^{\infty} \ln \left(1-e^{-\varepsilon / \Theta}\right) g_{0 f}(\varepsilon) d \varepsilon .
$$

In expressions (59), $(60), g_{0 e}(\varepsilon), g_{0 f}(\varepsilon)$ are given by formulas (39), (41), in which $G^{a a^{+}}(\varepsilon), G^{u u}(\varepsilon)$ are replaced by zero-order approximation Green's functions, $G_{0}^{a a^{+}}(\varepsilon), G_{0}^{u u}(\varepsilon)$.

Configuration component of the thermodynamic potential in the formula (58) depends on the distribution of atoms of different kinds and is defined as

$$
\Omega_{c}=\left\langle\Phi_{0}\right\rangle-\Theta S_{c},
$$

where $S_{c}=-\left\langle\ln P_{c}\right\rangle$-the configuration entropy, $P_{c}$-the distribution function of atoms over crystal lattice sites.

Configuration entropy can be represented as in Ref. [26]: 


$$
S_{c}=-\frac{1}{v}\left[\sum_{\lambda, i} P_{0 i}^{\lambda} \ln P_{0 i}^{\lambda}+\frac{1}{2} \sum_{\substack{\lambda, i \\ \lambda, l j \\(0 i) \neq(l j)}} P_{0 i l j}^{\lambda \lambda^{\prime}} \ln \frac{P_{0 i}^{\lambda \lambda^{\prime} l j}}{P_{0 i}^{\lambda} P_{l j}^{\lambda^{\prime}}}+\ldots\right] .
$$

Free energy $F$ as a function of volume $V$, temperature $T$, number of electrons $N_{e}$, and parameters of interatomic correlations $\left(\varepsilon_{n_{1} i_{1}, n_{2} i_{2}}, \eta\right)$ is determined by the thermodynamic potential $\Omega: F=\Omega+\mu_{e}\left\langle N_{e}\right\rangle$. In a weak dependence approximation of the mass operators on energy of electrons and phonons, free energy $F$ can be represented in the form [28]

$$
F=\Omega_{c}+\Omega_{e}+\Omega_{f}+\mu_{e}\langle Z\rangle,
$$

where $\Omega_{e}, \Omega_{f}$ are given by expressions (59), (60), in which $g_{0 e}(\varepsilon), g_{0 f}(\varepsilon)$ are replaced by $g_{e}(\varepsilon), g_{f}(\varepsilon)$ (see (39), (41)).

The values $F, \Omega_{c}, S_{c}, \Omega_{e}, \Omega_{f}$ in formulas (59)-(63) are calculated per one atom.

\section{ELECTRICAL CONDUCTIVITY}

To calculate the electrical conductivity tensor, we use the Kubo formula [33]:

$$
\sigma_{\alpha \beta}(\omega)=\int_{0}^{1 / \Theta} \int_{0}^{\infty} e^{i \omega t-\delta t}\langle\tilde{e}, \quad \sim \quad \hbar,
$$

where $J_{\alpha}$ is the operator of the $\alpha$-projection of the current density.

It follows from Eq. (64) that

$$
\operatorname{Re} \sigma_{\alpha \beta}(\omega)=\frac{i}{2 \omega}\left[G_{r}^{J_{\alpha} J_{\beta}}(\omega)-G_{a}^{J_{\alpha} J_{\beta}}(\omega)\right] .
$$

To calculate the spectral representations, $G_{r}^{J_{\alpha} J_{\beta}}(\omega)$ and $G_{a}^{J_{\alpha} J_{\beta}}(\omega)$, of the retarded and the advanced Green's functions, we use the expression for current-density operator,

$$
J_{\alpha}(t)=e \int \Psi^{+}(\xi, t) v_{\alpha} \Psi(\xi, t) d \xi,
$$

where $\Psi^{+}(\xi, t)$ and $\Psi(\xi, t)$ are the field operators of creation and annihilation of electron, respectively; $v_{\alpha}$ is the operator of the $\alpha$-projection of the velocity; $e$ is the electron charge. By integrating over $\xi$, we mean integrating over the crystal volume and summing over the projections of spin $\sigma$ onto the $z$-axis; the volume of the crystal is assumed equal to 
unity.

The temperature Green's function in this case is written as

$$
G^{J_{\alpha} J_{\beta}}\left(\tau, \tau^{\prime}\right)=\frac{e^{2}}{N V_{1}} \sum_{n_{1} n_{2} n_{3} n_{4}} v_{\alpha n_{4} n_{2}} v_{\beta n_{3} n_{1}} G^{\prime \prime}\left(n_{1} \tau^{\prime}, n_{2} \tau, n_{3} \tau^{\prime}, n_{4} \tau\right),
$$

where $V_{1}$-volume of primitive cell; the two-particle Green's function is written as

$$
G^{\prime \prime}\left(n_{1} \tau^{\prime}, n_{2} \tau, n_{3} \tau^{\prime}, n_{4} \tau\right)=\left\langle T_{\tau} a_{n_{1}}\left(\tau^{\prime}\right) a_{n_{2}}(\tau) a_{n_{3}}^{+}\left(\tau^{\prime}\right) a_{n_{4}}^{+}(\tau) \sigma(1 / \theta)\right\rangle_{0}\langle\sigma(1 / \theta)\rangle_{0}^{-1},
$$

$(n=n i \gamma)$. The two-particle Green's function (68) is described by the diagram in Fig. 6. Numbers in Fig. 6 correspond to point numbers; e.g., 1 corresponds to $\left(n_{1} i_{1} \gamma_{1} \tau_{1}\right)$.

Using the diagram technique for two-time temperature Green's function and neglecting the contributions of scattering processes on clusters of three or more sites, for the static conductivity tensor, we can get the expression:

$$
\begin{aligned}
& \sigma_{\alpha \beta}=\frac{e^{2} \hbar}{4 \pi} V_{1}{\underset{-\infty}{J}}_{-\infty} \frac{\partial f}{\partial \varepsilon_{1}} \sum_{s, s^{\prime}=+,-}\left(2 \delta_{s s^{\prime}}-1\right) \sum_{\sigma \gamma, i}\left\{v_{\beta} \tilde{I} \quad \begin{array}{l}
s^{\prime} \\
1
\end{array}\right)+ \\
& \left.+\sum_{\lambda} P_{0 i}^{\lambda} \tilde{1} \quad \sim \quad \text {; }\right)+ \\
& +\sum_{\lambda} P_{0 i}^{\lambda} \sum_{\substack{l j \neq 0 i, \lambda^{\prime}}} P_{l j 0 i}^{\lambda^{\prime} / \lambda}\left[\tilde{l} \quad \sim \quad\left(\varepsilon_{1}^{s^{\prime}}\right)+\right. \\
& +\tilde{1} \\
& +\tilde{I} \\
& +t_{l j}^{\lambda^{\prime}}\left(\varepsilon_{1}^{s}\right) \tilde{I} \\
& \left.+T^{(2) \lambda^{\prime} l j, \lambda 0 i}\left(\varepsilon_{1}^{s}\right) \tilde{l} \quad{ }_{1}^{s^{\prime}}\right) T^{(2) \lambda 0 i, \lambda^{\prime} l j}\left(\varepsilon_{1}^{s^{\prime}}\right)+
\end{aligned}
$$

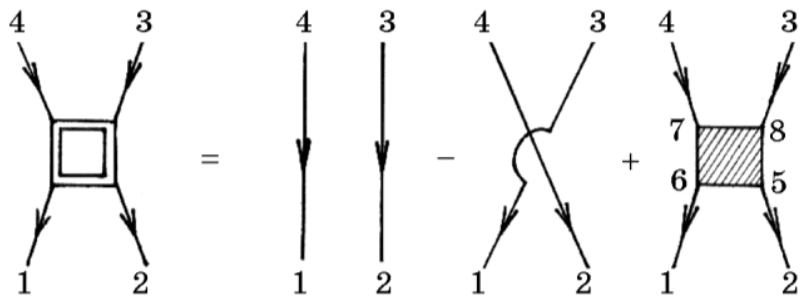

Fig. 6. Diagrams for the two-particle Green's function. 


$$
\begin{aligned}
& \left.\left.\left.\left.\left.+T^{(2) \lambda^{\prime} l j, \lambda 0 i}\left(\varepsilon_{1}^{s}\right) \tilde{I} \quad{ }_{1}^{s^{\prime}}\right) T^{(2) \lambda^{\prime} l j, \lambda 0 i}\left(\varepsilon_{1}^{s^{\prime}}\right)\right)\right]\right]\right\}^{0 i \gamma \sigma, 0 i \gamma \sigma}+ \\
& \left.+\int_{-\infty}^{\infty} \int_{-\infty}^{\infty} d \varepsilon_{1} d \varepsilon_{2} f\left(\varepsilon_{1}\right) f\left(\varepsilon_{2}\right)\left\langle\Delta G_{\alpha \beta}^{\mathrm{II}}\left(\varepsilon_{1}, \varepsilon_{2}\right)\right\rangle\right\},
\end{aligned}
$$

where $\tilde{i}$

$$
\text { , and }
$$

$$
\tilde{i} \quad \tilde{c}
$$

In formula (69), component $\Delta G_{\alpha \beta}^{\mathrm{II}}\left(\varepsilon_{1}, \varepsilon_{2}\right)$ of two-particle Green's function is caused by the interaction and has the form:

$$
\begin{gathered}
\Delta G_{\alpha \beta}^{\mathrm{II}}\left(\varepsilon_{1}, \varepsilon_{2}\right)=\frac{i}{2 \pi} v_{\alpha n_{4} n_{2}} v_{\beta n_{3} n_{1}}\left\{\left[G_{r n_{1} n_{6}}^{a a^{+}}\left(\varepsilon_{1}\right)-G_{a n_{1} n_{6}}^{a a^{+}}\left(\varepsilon_{1}\right)\right] \times\right. \\
\times\left[G_{r n_{2} n_{5}}^{a a^{+}}\left(\varepsilon_{2}\right)-G_{a n_{2} n_{5}}^{a a^{+}}\left(\varepsilon_{2}\right)\right]\left[G_{a n_{7} n_{4}}^{a a^{+}}\left(\varepsilon_{2}\right) G_{r n_{8} n_{3}}^{a a^{+}}\left(\varepsilon_{1}\right)-\right. \\
\left.-G_{r n_{7} n_{4}}^{a a^{+}}\left(\varepsilon_{2}\right) G_{a n_{8} n_{3}}^{a a^{+}}\left(\varepsilon_{1}\right)\right]+G_{a n_{1} n_{6}}^{a a^{+}}\left(\varepsilon_{1}\right)\left[G_{r n_{2} n_{5}}^{a a^{+}}\left(\varepsilon_{2}\right)-G_{a n_{2} n_{5}}^{a a^{+}}\left(\varepsilon_{2}\right)\right] \times \\
\times G_{a n_{7} n_{4}}^{a a^{+}}\left(\varepsilon_{2}\right)\left[G_{r n_{8} n_{3}}^{a a^{+}}\left(\varepsilon_{1}\right)-G_{a n_{8} n_{3}}^{a a^{+}}\left(\varepsilon_{1}\right)\right]-G_{r n_{1} n_{6}}^{a a^{+}}\left(\varepsilon_{1}\right) \times \\
\times\left[G_{r n_{2} n_{5}}^{a a^{+}}\left(\varepsilon_{2}\right)-G_{a n_{2} n_{5}}^{a a^{+}}\left(\varepsilon_{2}\right)\right] G_{r n_{7} n_{4}}^{a a^{+}}\left(\varepsilon_{2}\right)\left[G_{r n_{8} n_{3}}^{a a^{+}}\left(\varepsilon_{1}\right)-G_{a n_{8} n_{3}}^{a a+}\left(\varepsilon_{1}\right)\right]+ \\
+\left[G_{a n_{1} n_{6}}^{a a^{+}}\left(\varepsilon_{1}\right) G_{r n_{2} n_{5}}^{a a^{+}}\left(\varepsilon_{1}\right)-G_{r n_{1} n_{6}}^{a a^{+}}\left(\varepsilon_{1}\right) G_{a n_{2} n_{5}}^{a a^{+}}\left(\varepsilon_{1}\right)\right] \times \\
\left.\times\left[G_{r n_{1} n_{6}}^{a a n^{+}}\left(\varepsilon_{1}\right)-G_{a n_{7} n_{4}}^{a a^{+} n_{6}}\left(\varepsilon_{2}\right)\right] G_{a n_{7} n_{4}}^{a a n^{+}}\left(\varepsilon_{2}\right)\right]\left[G_{r n_{2} n_{5}}^{a a^{+}}\left(\varepsilon_{2}\right)\left[G_{r n_{7} n_{3}}^{a a^{+} n_{4}}\left(\varepsilon_{1}\right)-G_{a n_{8} n_{3}}^{a a^{+}}\left(\varepsilon_{1}\right)-G_{a n_{7} n_{4}}^{a a^{+}}\left(\varepsilon_{2}\right)\right] \times\right. \\
\times G_{r n_{8} n_{3}}^{a a^{+}}\left(\varepsilon_{1}\right)-\left[G_{r n_{1} n_{6}}^{a a^{+}}\left(\varepsilon_{1}\right)-G_{a n_{1} n_{6}}^{a a^{+}}\left(\varepsilon_{1}\right)\right] G_{a n_{2} n_{5}}^{a a^{+}}\left(\varepsilon_{2}\right) \times \\
\left.\times\left[G_{r n_{7} n_{4}}^{a a^{+}}\left(\varepsilon_{2}\right)-G_{a n_{7} n_{4}}^{a a^{+}}\left(\varepsilon_{2}\right)\right] G_{a n_{8} n_{3}}^{a a^{+}}\left(\varepsilon_{1}\right)\right\} \Gamma_{n_{5} n_{8}}^{n_{6} n_{7}}\left(\varepsilon_{1}, \varepsilon_{2} ; \varepsilon_{2}, \varepsilon_{1}\right)(n \equiv n i \gamma \sigma) .(
\end{gathered}
$$

Operator of $\alpha$-projection of the electron velocity in (69) is as follows:

$$
v_{\alpha i, i^{\prime}}(\mathbf{k})=\frac{1}{\hbar} h_{i, i^{\prime}}(\mathbf{k}) .
$$

To simplify the formula (69), we use approximate expression $\left\langle\Delta G_{\alpha \beta}^{\mathrm{II}}\left(\varepsilon_{1} ; \varepsilon_{2}\right)\right\rangle \approx \Delta \tilde{\boldsymbol{C}}$, where $\Delta \tilde{\boldsymbol{C}}$ is derived from the expression (70) by replacing $G^{a a^{+}}(\varepsilon)$ with $\tilde{c}$. 
It should be noted that the description of electron correlations in disordered crystals would have to come from Hamiltonian system that consists of interacting subsystems of nuclei and electrons. However, it leads to excessively large rank of the Hamiltonian matrix (1) and major complications in numerical calculations of electronic structure and properties of the system. To reduce the rank of the Hamiltonian matrix (1), the real wave functions of neutral free the $A$ kind atom are chosen as a basis.

Hamiltonian of the system consists of a single-electron Hamiltonian in the ion core field, the potential energy of the pair electron-electron interactions, Hamiltonian describing the vibrations of the lattice, and Hamiltonian of electron-phonon interaction.

The wave functions and potential energy of an electron in the $\lambda$ kind atom, which is located at the site $(0 i 1)$, are determined by the method of electron density functional from Kohn-Sham equation [4, 5, 6, 7].

Potential energy of electrons in the ion core and the Hamiltonian wave-function basis, we find from the Kohn-Sham equation for the noninteracting atoms [22, 23]:

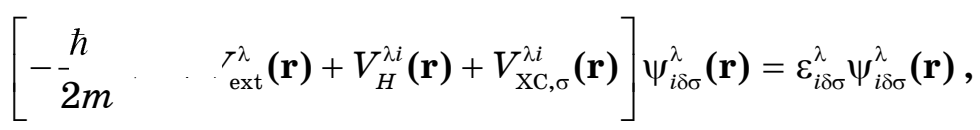

where $\sigma$-the quantum number of spin projection on the $z$-axis; $\delta=\boldsymbol{c}^{\sim} \quad ; l, m$-quantum numbers of angular momentum, and $\sim$ - the quantum number describing the value of electron energy. To reduce the record in the expression (71), $\left(\mathbf{r}-\mathbf{r}_{n i}\right)$ is denoted by $(\mathbf{r})$.

In expression (71), the value $V_{\text {ext }}^{\lambda}(\mathbf{r})$ is potential energy of an electron in the $\lambda$ kind atom core at the site $(n i)$;

$$
V_{H}^{\lambda i}(\mathbf{r})=\int d v^{\prime} \frac{e^{2}}{\left|\mathbf{r}-\mathbf{r}^{\prime}\right|} n_{\lambda i}\left(\mathbf{r}^{\prime}\right)
$$

is the Coulomb potential of the electron charge.

In formula (72), electron density is as follows:

$$
n_{\lambda i}(\mathbf{r})=n_{\lambda i \sigma}(\mathbf{r})+n_{\lambda i-\sigma}(\mathbf{r}) .
$$

Electron density with projection of spin $\sigma$ is given by expression

$$
n_{\lambda i \sigma}(\mathbf{r})=\sum_{\delta} Z_{i \delta \sigma}^{\lambda} \psi_{i \delta \sigma}^{\lambda *}(\mathbf{r}) \psi_{i \delta \sigma}^{\lambda}(\mathbf{r}),
$$

where $Z_{i \delta \sigma}^{\lambda}$-the occupation number of electron state $(i \delta \sigma)$, provided that the site ( $n i$ ) be occupied by the $\lambda$ kind atom, which is calculated by the formula (45). The expression (74) follows from the definition of the density operator of electrons [31] and the definition of the Green's 
function (15). In meta-generalized gradient approximation obtained by Perdew [6-10], based on the density functional theory of manyelectron systems, taking into account the spin polarization and inhomogeneous distribution of electron density, the result of the action of exchange-correlation potential $V_{\mathrm{XC}, \sigma}(\mathbf{r})=V_{\mathrm{XC}, \sigma}^{\mathrm{MGGA}}(\mathbf{r})$ on the wave function can be represented as follows:

$$
\begin{aligned}
& V_{\mathrm{XC}, \sigma}^{\mathrm{MGGA}}(\mathbf{r}) \psi_{\gamma \sigma}(\mathbf{r})=V_{\mathrm{XC}, \sigma}^{\mathrm{GGA}}(\mathbf{r}) \psi_{\gamma \sigma}(\mathbf{r})-\frac{1}{2} \nabla\left\{\mu_{\mathrm{XC}, \sigma}(\mathbf{r}) \nabla\right\} \psi_{\gamma \sigma}(\mathbf{r}), \\
& V_{\mathrm{XC}, \sigma}^{\mathrm{GGA}}(\mathbf{r})=\left[\frac{\partial e_{\mathrm{XC}}^{\mathrm{MGGA}}}{\partial n_{\sigma}}-\nabla\left(\frac{\partial e_{\mathrm{XC}}^{\mathrm{MGGA}}}{\partial \nabla n_{\sigma}}\right)\right], \mu_{\mathrm{XC}, \sigma}(\mathbf{r})=\frac{\partial e_{\mathrm{XC}}^{\mathrm{MGGA}}}{\partial \tau_{\sigma}},
\end{aligned}
$$

where $e_{\mathrm{XC}}^{\mathrm{MGGA}}\left(2 n_{\sigma}\right) / 2$ is the exchange-correlation energy density, $\tau_{\sigma}=\sum_{\delta}\left|\nabla \psi_{\delta \sigma}\right|^{2} / 2$ - kinetic energy density.

Wave functions of basis set, $\varphi_{n i \gamma \sigma}(\mathbf{r})$, on which Hamiltonian of the system is given by the set (1), are defined from Kohn-Sham equation (71) for the $\lambda=A$ kind atom and equal to $\left.\varphi_{n i \gamma \sigma}(\mathbf{r})=R_{i^{\sim}}^{A} \quad{ }^{\prime}-\mathbf{r}_{n i} \mid\right) Y_{l m}^{v}(\theta, \varphi)$, where $\left.R_{i^{\sim}}^{A} \quad{ }^{\prime}{ }_{--}-\mathbf{r}_{n i} \mid\right)$ - the radial part of wave function in Eq. (71), $\gamma=(\sigma v)$. Real spherical functions, $Y_{l m}^{v}(\theta, \varphi)$, are related to complex spherical functions, $Y_{l m}(\theta, \varphi)$, by relations

$$
Y_{l m}^{c}(\theta, \varphi)=\frac{Y_{l m}(\theta, \varphi)+Y_{l m}^{*}(\theta, \varphi)}{\sqrt{2}}, Y_{l m}^{s}(\theta, \varphi)=\frac{Y_{l m}(\theta, \varphi)-Y_{l m}^{*}(\theta, \varphi)}{i \sqrt{2}}(m \neq 0) .
$$

Electron density with spin projection $\sigma(74)$ can be represented as

$$
n_{\lambda i \sigma}(\mathbf{r})=n_{\lambda i \sigma}^{\prime}(\mathbf{r})+\Delta n_{\lambda i \sigma}(\mathbf{r})
$$

where

$$
n_{\lambda i \sigma}^{\prime}(\mathbf{r})=\sum_{\delta}{ }^{\prime} Z_{i \gamma \sigma}^{\lambda} \psi_{i \delta \sigma}^{\lambda *}(\mathbf{r}) \psi_{i \delta \sigma}^{\lambda}(\mathbf{r})
$$

is the density of electron states in the ion cores; summation is carried out over the filled states $Z_{i \delta \sigma}^{\lambda}=1$ of ion core, and

$$
\Delta n_{\lambda i \sigma}(\mathbf{r})=\sum_{\delta} Z_{i \delta \sigma}^{\lambda} \psi_{i \delta \sigma}^{\lambda *}(\mathbf{r}) \psi_{i \delta \sigma}^{\lambda}(\mathbf{r})
$$

is the density of electrons in valent states of the atom. We assume that the potential energy of electrons in the ion core and the Hamiltonian wave-function basis have the same form as for noninteracting atoms. 
This assumption puts a requirement that the value of occupation number $Z_{i \delta \sigma}^{\lambda}$ in expression (79) has the same value calculated by the formula (45).

Using formula (76), it can be shown that $Z_{i \delta \sigma}^{\lambda}$ in expression (79) is equal to

$$
Z_{i \delta \sigma}^{\lambda}=\frac{1}{2}\left(Z_{i \delta c \sigma}^{\lambda}+Z_{i \delta s \sigma}^{\lambda}\right)
$$

Here, $Z_{i \delta \mathrm{v} \sigma}^{\lambda}$ is calculated by expression (45), in which should be put $\gamma=(\sigma v)$, where $v=c, s$. Above-mentioned consistency can be achieved by using the iterative procedure, in which the value of the right side of expression (80), which is calculated by the formula (45), at a certain step of iteration with a given accuracy coincides with the left side of expression (80) in the previous step.

Matrix elements $h_{n_{1} i_{1}, n_{2} i_{\gamma_{2}}}^{(0)}$ of Hamiltonian (1) in multi-band $s-p-d$ tight-binding model are the matrix elements of the operator of kinetic energy and potential energy of an electron in a potential of the ion core, ${ }^{i}{ }^{\prime} L_{n i}{ }^{i i}\left(\mathbf{r}-\mathbf{r}_{n i}\right)$, on the real wave functions of the neutral $A$ kind atoms. Potential energy of an electron in the field of ion core, $v^{A n i}(\mathbf{r})$, is determined by formulas (76)-(79), in which, instead of the total electron density, one should put the density of electrons, $n_{A i \sigma}^{\prime}(\mathbf{r})$ (79), in states of the $A$ kind ion core.

Diagonal (by site number $\left(n_{1} i_{1}\right)$ ) matrix elements are equal to

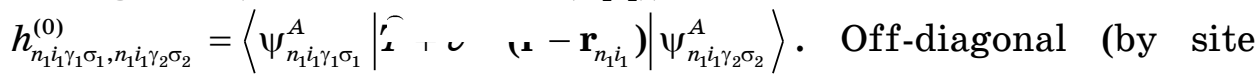
number) matrix elements can be presented in the following form:

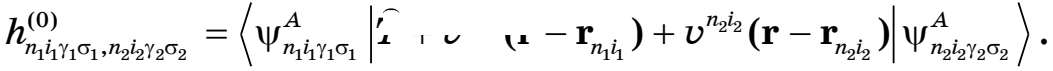

Matrix elements in the right side of the last formula are calculated by the Slater-Koster method [18, 19].

Potential energy of an electron in the ion core field in the expression (11) is determined by formulas (76)-(78), in which, instead of the total electron density, the density of electrons, $n_{\lambda i \sigma}^{\prime}(\mathbf{r})(78)$, in states of the $A$ kind ion core should put.

Matrix elements $v_{n_{3} j_{3}, n_{4} i_{4} \gamma_{4}}^{(2) n_{1} \gamma_{1}, n_{2} i_{2}}$ of Hamiltonian (1) can be calculated by integrating the angular variables. Integral of the product of three spherical functions (Gaunt integral) can be represented using the Clebsch-Gordan coefficients [34]. As a result, for matrix elements $v_{l_{3} m_{3}, l_{4} m_{4}}^{(2) l_{1} m_{1}, m_{2} m_{2}}$, we obtain 


$$
\begin{gathered}
v_{l_{3} m_{3}, l_{4} m_{4}}^{(2) l_{2} m_{1}, l_{2} m_{2}}=e^{2} \sum_{\substack{l=0 \\
\left|l-l^{\prime} \leq l_{3} \leq l+l^{\prime}\\
\right| l_{2}-h_{1} \mid \leq l_{3} \leq l_{2}+l_{1} \\
l+l^{\prime} l_{3}=2 \prime^{\prime}, n^{\prime}=0,1,2,3, \ldots \\
l_{2}+l_{1}+l_{3}=2 n_{1}, n_{1}=0,1,2,3, \ldots}} \frac{1}{2 l_{3}+1}\left[\frac{\left(2 l_{3}+1\right)\left(2 l^{\prime}+1\right)\left(2 l_{3}+1\right)\left(2 l_{1}+1\right)}{(2 l+1)\left(2 l_{2}+1\right)}\right]^{1 / 2} \times \\
\times c\left(l_{3} l^{\prime} l ; 0,0\right) c\left(l_{3} l^{\prime} l ; m^{\prime}-m, m^{\prime}\right) c\left(l_{3} l_{1} l_{2} ; 0,0\right) c\left(l_{3} l_{1} l_{2} ; m_{2}-m_{1}, m_{1}\right) \times \\
{\left[\int_{0}^{\infty} d r_{1} r_{1}^{2} R_{n l}\left(r_{1}\right) R_{n^{\prime} l^{\prime}}\left(r_{1}\right) \int_{0}^{r_{1}} d r_{2} r_{2}^{2} R_{n_{2} l_{2}}\left(r_{2}\right) R_{n_{1} l_{1}}\left(r_{2}\right) \frac{r_{2}^{l_{3}}}{r_{1}^{l_{3}+1}}+\right.} \\
\left.+\int_{0}^{\infty} d r_{2} r_{2}^{2} R_{n_{2} l_{2}}\left(r_{2}\right) R_{n_{1} l_{1}}\left(r_{2}\right) \int_{0}^{r_{2}} d r_{1} r_{1}^{2} R_{n l}\left(r_{1}\right) R_{n^{\prime} l^{\prime}}\left(r_{1}\right) \frac{r_{1}^{l_{3}}}{r_{2}^{l_{3}+1}}\right]
\end{gathered}
$$

where $l, m$ are orbital and magnetic quantum numbers, respectively, $c\left(l^{\prime \prime} l^{\prime} l ; m^{\prime \prime}, m^{\prime}\right)$ - the Clebsch-Gordan coefficients [34], $R_{n l}(r)$-the radial part of wave function, $n$ - the main quantum number.

Matrix elements on the basis of real wave functions [18], $v_{n_{3} i_{3} \gamma_{3}, n_{4} i_{4} \gamma_{4}}^{(2) n_{1} \gamma_{1}, n_{2} i_{2} \gamma_{2}}$, for each site are expressed by linear combinations of matrix elements $v_{l_{3} m_{3}, l_{4} m_{4}}^{(2) m_{2} m_{2} m_{2}}$. This calculation procedure for matrix elements can be easily programmed.

Matrix elements on the basis of real wave functions, $v_{n_{3} i_{3} \gamma_{3}, n_{4} i_{4} \gamma_{4}}^{(2) n_{1} \gamma_{1}, n_{2} \gamma_{2}}$, for different sites can be approximately represented in the form similar to formula (81), if we describe radial part of the wave function by Gaussian function (Gaussian orbital) as this is done in the method of molecular orbitals-linear combinations of atomic orbitals [35]. In this approximation, the multicentre integrals $v_{n_{3} i_{3} \gamma_{3}, n_{4} i_{4} \gamma_{4}}^{\left(2 n_{1} i_{1} \gamma_{1}, n_{2} \gamma_{2}\right.}$ have the form of one-centre integrals, as the product of two Gaussian orbitals, which are localized at different centres, can be reduced to the product of orbitals, which are localized at the joint centre.

To investigate the influence of an external magnetic field on the energy spectrum of electrons and electrical conductivity member describing the additive energy of the crystal in an external magnetic field, it is need to be added to Hamiltonian of the system $H(1)$ :

$$
H^{\prime}=\sum_{n_{1} \gamma_{1} \sigma_{1}} 2 \mu_{B} \sigma_{1} \mathrm{H} a_{n_{1} \gamma_{1} \gamma_{1} \sigma_{1}}^{+} a_{n_{1} i_{1} \gamma_{1} \sigma_{1}},
$$

where $\mu_{B}, \mathrm{H}, \sigma_{1}$ are the Bohr magneton, intensity of external magnetic field, and electron spin projection on the direction of the magnetic field, respectively. 
Spin-dependent transport in systems with strong electron correlations is described by partial constituent of diagonal conductivity tensor element (79), which corresponds to a specific value of the electron spin projection.

Thus, in the obtained expressions for the Green's functions of electrons and phonons, free energy and electrical conductivity of the crystal, the processes of electron scattering on the potential of ion core, charge and spin fluctuations of density and lattice vibrations are taken into account. The accuracy of calculating the electronic structure, vibrational spectra and electrical conductivity of the crystal is determined by precision of vertex renormalization parts of the mass operators of electron-electron and electron-phonon interactions and the small parameter of the cluster expansion for the Green's functions of electrons and phonons.

\section{RESULTS OF CALCULATIONS AND CONCLUSIONS}

Here are the results of calculation of the energy spectrum of electrons and phonons and conductivity of carbon nanotubes doped with chromium. In calculation, renormalization of vertex parts of mass operator of electron-electron and electron-phonon interactions (21)-(23) was neglected. Real wave functions of $2 s-, 2 p$-, $3 s$-, $3 d$-states of neutral noninteracting atoms of carbon were chosen. In the above-mentioned selfconsistent iterative procedure of calculation of electronic structure and free energy, in the first step of the iteration formula (44), $Z_{i_{\gamma \gamma \sigma}}^{\lambda}$ were put equal to occupation numbers of the corresponding electron states of neutral noninteracting atoms of carbon and $\mathrm{Cr}$. The off-diagonal (by site index (ni)) matrix elements of Hamiltonian (1) were calculated by taking into account the first three coordination spheres. Contribution to the static displacements of atoms was neglected in the calculations. Calculations were performed for the temperature $T=300 \mathrm{~K}$.

We performed geometry optimization of the crystal structure of carbon nanotube of chirality $(3,0)$ with $\mathrm{Cr}$ impurity. Geometric optimization of the crystal structure was achieved by minimizing the free energy (32). Carbon nanotube doped with $\mathrm{Cr}$ has a one-dimensional crystal structure. Primitive cell contains 18 non-equivalent atom positions. Carbon atoms are located in 12 positions on the surface of the inner cylinder. The distance between the carbon atoms is $0.142 \mathrm{~nm}$. The $\mathrm{Cr}$ atoms are randomly located in six positions on the outer surface of the cylinder opposite the centre of a hexagon, the vertices of which are carbon atoms. Through the study of free energy minimum, it is found that $\mathrm{Cr}$ atoms are randomly located on the surface of nanotubes. This indicates that the probability of $\mathrm{Cr}$ atoms arrangement in the expression (51) $P_{l j 0 i}^{\lambda^{\prime} / \lambda}=P_{l j}^{\lambda^{\prime}}=c^{\lambda^{\prime}}$, where $c^{\lambda^{\prime}}$ - the ratio of $\mathrm{Cr}$ atoms with re- 
spect to the six possible positions of the atoms $\mathrm{Cr}$ within the primitive cell. The distance between carbon atoms and $\mathrm{Cr}$ is $0.22 \mathrm{~nm}$. The relative position of carbon atoms and $\mathrm{Cr}$ is similar to the location of atoms of transition metals on the surface of carbon nanotubes of large diameter, which are described in [35] by ultrasoft pseudopotential method.

The value of localized magnetic moment projection of the $\mathrm{Cr}$ atom and induced localized magnetic moment of the $\mathrm{C}$ atom in the direction of the magnetic field increases with the intensity of field. For carbon nanotubes with $5 \mathrm{Cr}$ atoms in primitive cell, value of projection magnetic moment of the Cr atom varies as $m_{\mathrm{Cr}}=(1.02 ; 2.24) \mu_{B}$, and the magnetic moment of the $\mathrm{C}$ atom varies as $m_{\mathrm{C}}=(0.0036 ; 0.02) \mu_{B}$ with increasing values of the magnetic field intensity from zero to $\mathrm{H}=200 \mathrm{~A} / \mathrm{m}$.

As calculated by means of the formula (39), Figure 7 shows the partial $\left(g_{e \sigma}(\varepsilon)=\frac{1}{v} \sum_{i, \gamma, \lambda} P_{0 i}^{\lambda} g_{0 i \gamma \sigma}^{\lambda}(\varepsilon)\right)$ and total $\left(g_{e}(\varepsilon)=\sum_{\sigma} g_{e \sigma}(\varepsilon)\right)$ electron densities of states of carbon nanotube with an admixture of $\mathrm{Cr}$ in the absence of external magnetic field. Vertical line shows the Fermi level $\varepsilon_{F}$.

In Figure 8, points show the dependence of free energy $F(32)$ on the parameter of pair correlations in arrangement of $\mathrm{Cr}$ impurities on lattice sites, $\varepsilon^{B B}=\varepsilon_{l j 0 i}^{B B}(54)$ for the first coordination sphere (atom of $\mathrm{Cr}$ is denoted as the $B$ kind atom). The dependence $F\left(\varepsilon^{B B}\right)$ is shown in the region of free energy minimum. The free energy is measured from average electrostatic interaction energy of the $A$ kind ions, $\left\langle\Phi_{0}\right\rangle(61)$.

Figure 9 shows the partial $\left(g_{e \sigma}(\varepsilon)\right)$ and total $\left(g_{e}(\varepsilon)\right)$ electron densities of states of carbon nanotube with five atoms of $\mathrm{Cr}$ per primitive cell in external magnetic field of $\mathrm{H}=100 \mathrm{~A} / \mathrm{m}$. In Figure 9, the part of the energy spectrum that is close to the Fermi level is shown. As shown in

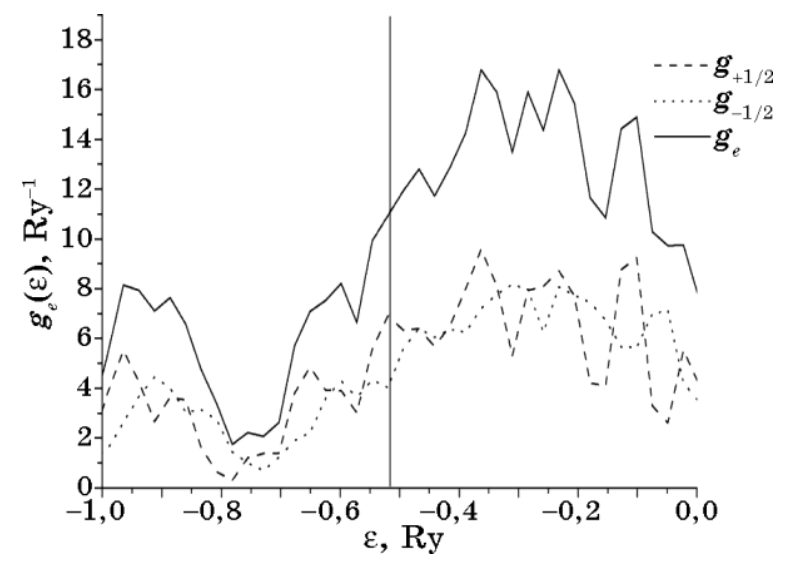

Fig. 7. Electron densities of states of carbon nanotube with an admixture of $\mathrm{Cr}$. 


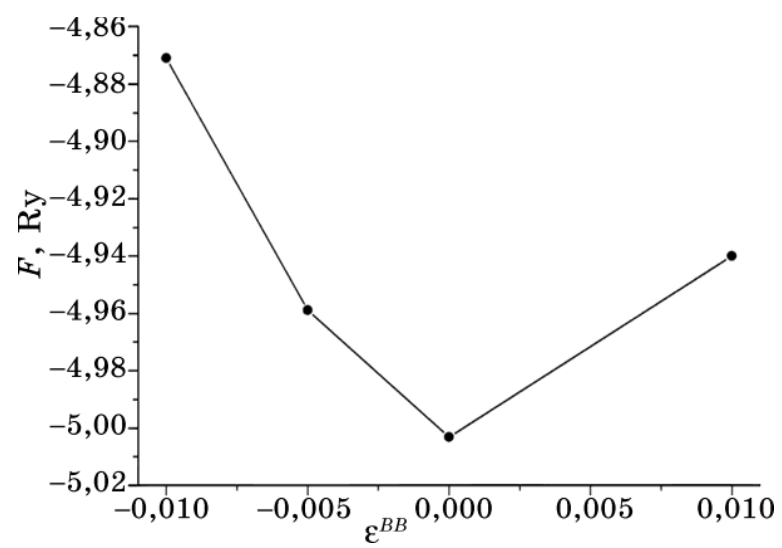

Fig. 8. Dependence of free energy $F$ for carbon nanotubes with five atoms of Cr per primitive cell on the parameter of pair correlations in arrangement of Cr impurities on lattice sites, $\varepsilon^{B B}$.

Figure 9, for a given sign of the projection of magnetic moment localized on sites of lattice, energies of the electron with spin $\sigma=1 / 2$ are shifted relative to values of the energy of the electron with spin $\sigma=-1 / 2$. This leads to the formation of the Coulomb gap in the energy spectrum of electrons, which is visible on $g_{\frac{1}{1} / 2}(\varepsilon)$ and on $g(\varepsilon)$ of Fig. 9.

The results presented in Fig. 9 are qualitatively consistent with results obtained by another method in Ref. [37].

In Figure 10, the dependence of the spin polarized electric current,

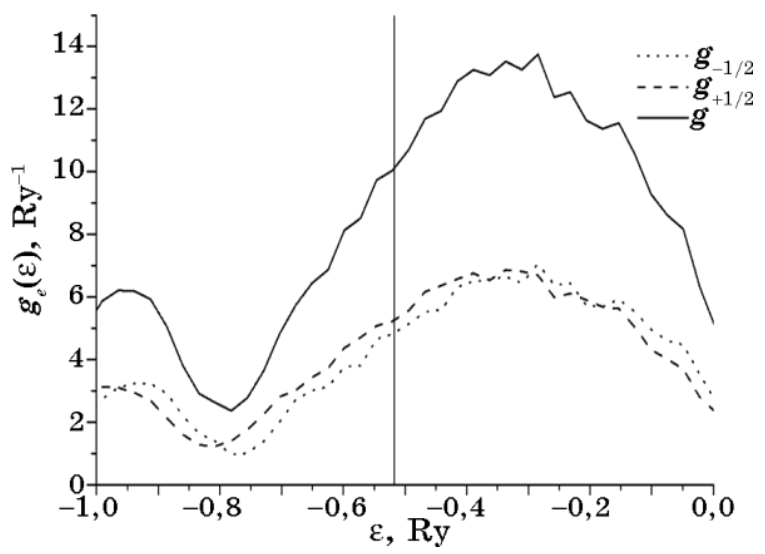

Fig. 9. Electron densities of states of carbon nanotube with five atoms of $\mathrm{Cr}$ per primitive cell in external magnetic field of $\mathrm{H}=100 \mathrm{~A} / \mathrm{m}$. 


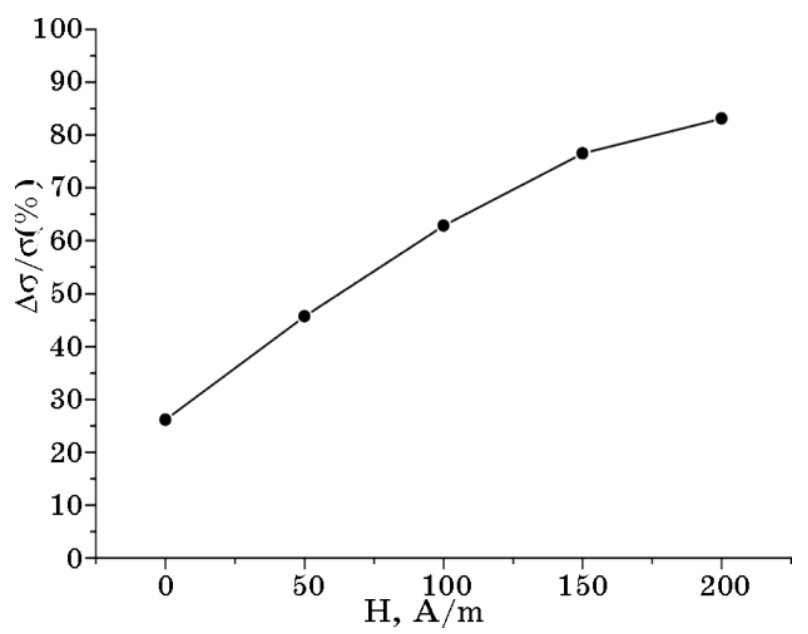

Fig. 10. The dependence of spin polarized electric current, $\Delta \sigma / \sigma$, in carbon nanotube on the magnitude of the external magnetic field, $\mathrm{H}$.

$\Delta \sigma / \sigma=\left(\sigma_{1 / 2}-\sigma_{-1 / 2}\right) / \sigma$, in carbon nanotube with chirality $(3,0)$ on the magnitude of the external magnetic field as calculated by the formula (37) for temperature of $300 \mathrm{~K}$ is shown.

Thus, the phenomenon of spin-dependent electron transport occurs in systems with strong electron correlations and is associated with the location of the Fermi level relative to the Coulomb gap in the energy spectrum of electrons.

In an external magnetic field, there is relative displacement of electrons' energy levels (Coulomb gap) for different spin projections $\sigma$ (Fig. 9).

Value of spin polarization of electron transport depends on the difference of electron density states with different spin projection at the Fermi level.

In Ref. [38], the electronic structure and properties of carbon nanotubes with transition metal chains adsorbed on the surface is calculated, based on the density functional method using ultrasoft pseudopotential. Our results are qualitatively consistent with the results of [38], in which $a b$ initio electron density functional method shows that the chains of transition metals adsorbed on the surface of carbon nanotubes open a gap in the electrons' states with a certain spin value.

\section{REFERENCES}

1. W. A. Harrison, Pseudopotentials in the Theory of Metals (New York: Benja$\min : 1966)$. 
2. D. Vanderbilt, Phys. Rev. B, 41, No. 11: 7892 (1985).

3. K. Laasonen, R. Car, C. Lee, and D. Vanderbilt, Phys. Rev. B, 43, No. 8: 6796 (1991).

4. P. E. Blochl, Phys. Rev. B, 50, No. 24: 17953 (1994).

5. G. Kresse and D. Joubert, Phys. Rev. B, 59, No. 3: 1758 (1999).

6. J. P. Perdew, K. Burke, and M. Ernzerhof, Phys. Rev. Lett., 77, No. 18: 3865 (1996).

7. J. Tao, J. P. Perdew, V. N. Staroverov, and G. E. Scuseria, Phys. Rev. Lett., 91, No. 14: 146401 (2003).

8. J. P. Perdew, S. Kurth, A. Zupan, and P. Blaha, Phys. Rev. Lett., 82, No. 12: 2544 (1999).

9. J. P. Perdew, A. Ruzsinszky, G. I. Csonka, L. A. Constantin, and J. Sun, Phys. Rev. Lett., 103, No. 2: 026403 (2009); ibidem, 106, No. 17: 179902 (2011).

10. J. Sun, M. Marsman, G. I. Csonka, A. Ruzsinszky, P. Hao, Y.-S. Kim, G. Kresse, and J. P. Perdew, Phys. Rev. B, 84, No. 3: 035117 (2011).

11. V. V. Ivanovskaya, C. Köhler, and G. Seifert, Phys. Rev., 75, No. 7: 075410 (2007).

12. D. Porezag, T. Frauenheim, T. Köhler, G. Seifert, and R. Kascher, Phys. Rev. B, 51, No. 19: 12947 (1995).

13. M. Elstner, D. Porezag, G. Jungnickel, J. Elsner, M. Haugk, Th. Frauenheim, S. Suhai, and G. Seifert, Phys. Rev. B, 58, No. 11: 7260 (1998).

14. C. Köhler, G. Seifert, U. Gerstmann, M. Elstner, H. Overhof, and Th. Frauenheim, Phys. Chem. Chem. Phys., 3, No. 23: 5109 (2001).

15. V. V. Ivanovskaya and G. Seifert, Solid State Commun., 130, No. 3-4: 175 (2004).

16. V. V. Ivanovskaya, T. Heine, S. Gemming, and G. Seifert, phys. status solidi (b), 243, No. 8: 1757 (2006).

17. A. Enyaschin, S. Gemming, T. Heine, G. Seifert, and L. Zhechkov, Phys. Chem. Chem. Phys., 8, No. 28: 3320 (2006).

18. J. C. Slater and G. F. Koster, Phys. Rev., 94, No. 6: 1498 (1954).

19. R. R. Sharma, Phys. Rev. B, 19, No. 6: 2813 (1979).

20. J. B. Staunton, S. S. A. Razee, M. F. Ling, D. D. Johnson, and F. J. Pinski, J. Phys. D: Appl. Phys., 31, No. 19: 2355 (1998).

21. S. S. A. Razee, J. B. Staunton, B. Ginatempo, E. Bruno, and F. J. Pinski, J. Phys.: Condens. Matter, 13, No. 38: 8565 (2001).

22. W. Kohn and L. J. Sham, Phys. Rev., 140, No. 4A: 1133 (1965).

23. R. O. Jones and O. Gunnarsson, Rev. Mod. Phys., 61, No. 3: 689 (1989).

24. G. M. Stocks, W. M. Temmerman, and B. L. Gyorffy, Phys. Rev. Lett., 41, No. 5: 339 (1978)

25. G. M. Stocks, and H. Winter, Z. Phys. B, 46: 95 (1982).

26. D. D. Johnson, D. M. Nicholson, F. J. Pinski, B. L. Gyorffy, and G. M. Stocks, Phys. Rev. B, 41, No. 14: 9701 (1990).

27. R. V. Chepulskii and W. H. Butler, Phys. Rev. B, 72, No. 13: 134205 (2005).

28. S. P. Repetsky and T. D. Shatnii, Theor. Math. Phys., 131, No. 3: 832 (2002).

29. A. A. Abrikosov, L. P. Gorkov, and I. E. Dzyaloshinski, Methods of Quantum Field Theory in Statistical Physics (Eds. R. A. Silverman) (Englewood Cliffs, New Jersey: Prentice-Hall: 1963).

30. D. N. Zubarev, Nonequilibrium Statistical Thermodynamics (Eds. P. Gray and P. J. Shepherd) (New York: Consultants Bureau: 1974). 
31. F. Ducastelle, J. Phys. C, 7, No. 10: 1795 (1974).

32. V. F. Los' and S. P. Repetsky, J. Phys.: Condens. Matter, 6, No. 9: 1707 (1994).

33. R. Kubo, J.Phys. Soc. Japan, 12, No. 6: 570 (1957).

34. E. P. Wigner, Group Theory (New York-London: Academic Press: 1959).

35. J. C. Slater, Quantum Theory of Molecules and Solids. Vol. 1 (New York: McGraw-Hill: 1963).

36. C. Yang, J. Zhao, and J. P. Lu, Phys. Rev. Lett., 90, No. 25: 257203 (2003).

37. C. Yang, J. Zhao, and J. P. Lu, Nano Lett., 4, No. 4: 561 (2004).

38. E. Durgun and S. Ciraci, Phys. Rev. B, 74, No. 12: 125404 (2006). 